Article

\title{
Revisiting the Aluminum Trimesate-based MOF (MIL-96): from Structure Determination to the Processing of Mixed Matrix Membranes for CO2 Capture.
}

Marvin Benzaqui, Renjith S. Pillai, Anahid Sabetghadam, Virginie Benoit, Périne Normand, Jerome Marrot, Nicolas Menguy, David Montero, William Shepard, Antoine Tissot, Charlotte Martineau-Corcos, Clémence Sicard, Mihail Mihaylov, Florent Carn, Isabelle Beurroeis, Philip L. Llewellyn, Guy De Weireld, Konstantin Hadjiivanov, Jorge Gascon, Freek Kapteijn, Guillaume Maurin, Nathalie Steunou, and Christian Serre Chem. Mater., Just Accepted Manuscript • DOI: 10.1021/acs.chemmater.7b03203 • Publication Date (Web): 08 Nov 2017 Downloaded from http://pubs.acs.org on November 21, 2017

\section{Just Accepted}

"Just Accepted" manuscripts have been peer-reviewed and accepted for publication. They are posted online prior to technical editing, formatting for publication and author proofing. The American Chemical Society provides "Just Accepted" as a free service to the research community to expedite the dissemination of scientific material as soon as possible after acceptance. "Just Accepted" manuscripts appear in full in PDF format accompanied by an HTML abstract. "Just Accepted" manuscripts have been fully peer reviewed, but should not be considered the official version of record. They are accessible to all readers and citable by the Digital Object Identifier (DOI®). "Just Accepted" is an optional service offered to authors. Therefore, the "Just Accepted" Web site may not include all articles that will be published in the journal. After a manuscript is technically edited and formatted, it will be removed from the "Just Accepted" Web site and published as an ASAP article. Note that technical editing may introduce minor changes to the manuscript text and/or graphics which could affect content, and all legal disclaimers and ethical guidelines that apply to the journal pertain. ACS cannot be held responsible for errors or consequences arising from the use of information contained in these "Just Accepted" manuscripts. 
Revisiting the Aluminum Trimesate-based MOF (MIL-96): from Structure Determination to the Processing of Mixed Matrix Membranes for $\mathrm{CO}_{2}$ Capture.

Marvin Benzaqui, ${ }^{\dagger, \|}$ Renjith S Pillai, ${ }^{\dagger}$ Anahid Sabetghadam, ${ }^{\S}$ Virginie Benoit, $\perp$ Perine Normand, ${ }^{\partial}$

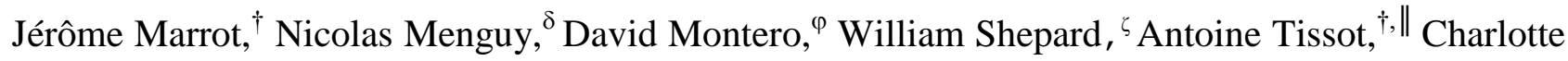
Martineau-Corcos, ${ }^{\dagger, \#}$ Clémence Sicard,$^{\dagger}$ Mihail Mihaylov, ${ }^{\Delta}$ Florent Carn,${ }^{\Upsilon}$ Isabelle Beurroies, ${ }^{\perp}$ Philip L. Llewellyn, ${ }^{\perp}$ Guy De Weireld,${ }^{\partial}$ Konstantin Hadjiivanov, ${ }^{\Delta}$ Jorge Gascon,,${ }^{\S}$, Freek Kapteijn, ${ }^{\S}$ Guillaume Maurin, ${ }^{\ddagger}$ Nathalie Steunou, ${ }^{\dagger *}$ Christian Serre ${ }^{\dagger}, \|$

${ }^{\dagger}$ Institut Lavoisier de Versailles, UMR CNRS 8180, Université de Versailles St Quentin en Yvelines, Université Paris Saclay, 45 avenue des Etats-Unis 78035 Versailles Cedex. France.

"Institut des Matériaux Poreux de Paris, FRE 2000 CNRS, Ecole Normale Supérieure, Ecole Supérieure de Physique et de Chimie Industrielle de Paris, PSL Research University, 75005 Paris, France.

$\$$ Institut Charles Gerhardt Montpellier, UMR 5253 CNRS, Université de Montpellier, Place E. Bataillon, 34095 Montpellier Cedex 05, France.

$\S$ Catalysis Engineering-Chemical Engineering Department, Delft University of Technology, Van der Maasweg 9, 2629 HZ Delft, The Netherlands.

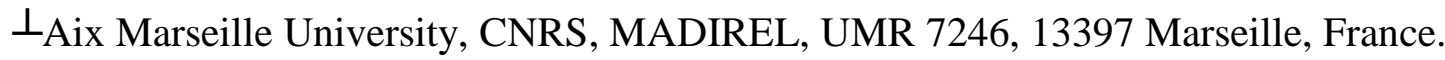

${ }^{\partial}$ Service de Thermodynamique et de Physique mathématique, Faculté Polytechnique, Université de Mons, 20 Place du Parc , 7000 Mons, Belgique.

${ }^{\delta}$ Institut de Minéralogie de Physique des Matériaux et de Cosmochimie, UMR 7590 CNRS UPMC Université Paris 06 MNHN IRD - Sorbonne Universités, 4 place Jussieu, 75005 Paris, France.

${ }^{\varphi}$ Institut des Matériaux de Paris Centre (IMPC), FR 2482 CNRS UPMC-Sorbonne Universités, 4 place Jussieu, 75252 Paris cedex 05, France.

${ }^{\zeta}$ Synchrotron Soleil, L’Orme des Merisiers, Saint Aubin, BP 48, 91192 Gif-sur-Yvette, France.

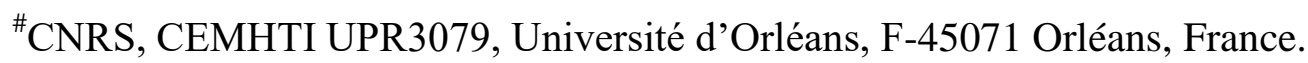

${ }^{\Delta}$ Institute of General and Inorganic Chemistry, Bulgarian Academy of Sciences, Sofia 1113, Bulgaria. 
${ }^{\Upsilon}$ Laboratoire Matière et Systèmes Complexes (MSC), UMR CNRS 7057, Université Paris Diderot, Bât. Condorcet, 10 rue A. Domon et L. Duquet, 75013 Paris, France.

${ }^{\Phi}$ King Abdullah University of Science and Technology, KAUST Catalysis Center, Advanced Catalytic Materials. Thuwal 23955, Saudi Arabia.

\begin{abstract}
A microporous Al trimesate-based Metal Organic Framework (MOF), denoted MIL-96(Al), was selected as a porous hybrid filler for the processing of Mixed Matrix Membranes (MMMs) for $\mathrm{CO}_{2} / \mathrm{N}_{2}$ post combustion separation. First, the structural model of MIL-96(Al) initially reported was revisited using a combination of synchrotron-based single crystal X-ray diffraction (XRD), solid state Nuclear Magnetic Resonance (NMR) spectroscopy and Density Functional Theory (DFT) calculations. In a second step, pure MIL-96 (Al) crystals differing by their size and aspect ratio, including anisotropic hexagonal platelets and nanoparticles of about $70 \mathrm{~nm}$ in diameter, were prepared. Then, a combination of in situ IR spectroscopy, single gas and $\mathrm{CO}_{2} / \mathrm{N}_{2}$ co-adsorption experiments, calorimetry and molecular simulations revealed that MIL-96(Al) nanoparticles show a relatively high $\mathrm{CO}_{2}$ affinity over $\mathrm{N}_{2}$ owing to strong interactions between $\mathrm{CO}_{2}$ molecules and several adsorption sites such as $\mathrm{Al}^{3+}$ Lewis centers, coordinated water and hydroxyl groups. Finally, the high compatibility between MIL-96(Al) nanoparticles and the 6FDA-DAM polymer allowed the processing of homogeneous and defect-free MMMs with a high MOF loading (up to $25 \mathrm{wt} \%$ ) that outperform pure polymer membranes for $\mathrm{CO}_{2} / \mathrm{N}_{2}$ separation.
\end{abstract}

Keywords: MOF, Polymer, Aluminum, MIL-96(Al), mixed matrix membrane, $\mathrm{CO}_{2}$ capture.

\title{
— INTRODUCTION
}

Metal Organic Frameworks (MOFs) have developed rapidly due to their outstanding properties and myriad of potential applications such as gas storage, separations, catalysis, biomedicine, electronic devices, and information storage among others. ${ }^{1,2}$ These hybrid porous crystalline materials consist of periodic coordination networks composed of inorganic building blocks and organic multifunctional ligands and can be rationally designed through crystal engineering. The explosion 
of interest in this class of materials stems from their endless chemical variability and unprecedented diversity of pore structure (shape and diameter of pores) and topology (two- to three-periodic extended frameworks), enabling the fine-tuning of their physico-chemical features for a targeted application. ${ }^{1,2,3,4}$ As a result of their crystallinity, these materials are well suited to the in-depth characterization of their structure as well as establishing clear structure-property relationships., 5 However, the synthesis and/or structure determination of numerous MOFs remains a hurdle particularly due to the high reactivity of their precursors and thus their low crystallinity. ${ }^{7}$ In addition, the complexity of their architecture associated with a possible structural disorder (water molecules, guest molecules, free ions, defects,...) and more importantly the existence of crystals with large unit cells and low symmetry have led to the development of methodologies that combine X-ray or neutron diffraction to advanced complementary experimental (solid state NMR, EXAFS, transmission electron microscopy...) and computational tools. ${ }^{8}$ For instance, solid-state NMR experiments integrating high magnetic field, specific pulse sequences, multiple-resonance decoupling and ultrafast magic-angle spinning (MAS) has become a powerful tool to provide information on the local environment of atoms (symmetry, coordination shell...) and probe their spatial proximity and connectivity, thereby constraining the possible structural solutions.,10 Moreover, modelling tools involving energy minimization techniques at the force field (interatomic potential) and/or electronic (quantum) levels were combined with experimental information for the structure resolution of numerous MOFs with low symmetry and/or poor crystallinity. ${ }^{8,11,12,13,14}$

Here, this paper intends to reinvestigate the crystallographic structure of the microporous aluminum trimesate MIL-96(Al), ${ }^{15}$ with a subtle combination of advanced experimental (X-ray diffraction, Solid State NMR, Infra-red spectroscopy) techniques and Density Functional Theory calculations. The periodic aluminum-oxo framework including the coordination sphere of $\mathrm{Al}$ centers was carefully determined. Moreover, the location of water molecules and hydroxo groups has allowed the description of the hydrogen-bond network in the porosity of the material. Narrow pores MOFs are a sub-class of porous materials of special interest owing to their pore aperture sizes below 4-7 $\AA$ that can potentially address key challenges such as carbon capture and 
separation of olefin/paraffin, acetylene/ethylene, linear/branched alkanes, xenon/krypton. ${ }^{16}$ The separation ability of MIL-96(Al) was previously demonstrated for several relevant applications of interest. ${ }^{17,18,19,20,21}$ Moreover, its rigid framework with relative small pores incorporating diverse potential adsorption sites ( $\mathrm{Al}$ acid sites, hydroxyl groups and coordinating water) makes this MOF attractive for the selective capture of $\mathrm{CO}_{2}$ over $\mathrm{N}_{2}$. This has been here further confirmed by a subtle combination of single component $\mathrm{CO}_{2}$ and $\mathrm{N}_{2}$ and their binary mixture adsorption measurements and grand canonical Monte Carlo simulations.

Due to its high thermal and hydrothermal stability, MIL-96(Al) was further envisaged for the processing of mixed matrix membrane (MMM) for post-combustion $\mathrm{CO}_{2}$ capture. These composite membranes which consist of filler particles dispersed into an organic polymer phase potentially combine the gas transport and separation properties of the incorporated particles with the good processability and mechanical properties of the polymers. Indeed, MOFs were recently proposed as fillers due to their outstanding gas separation properties. ${ }^{5}$ However, MOF-based MMMs suffer from several limitations mainly related to a possible physico-chemical mismatch between MOFs and polymers. ${ }^{22,23,24,25,26}$ Such a lack of chemical compatibility between both components limits the MOF loading of numerous MMMs and thus their performance. ${ }^{22-26}$ Indeed, for numerous MOF based MMMs, the transport properties and thus the selectivity of the membrane are driven by the polymer matrix which is the dominant component. In addition, a poor dispersion of MOFs fillers in the polymer matrix may take place thereby creating interphase defects (macro or nanovoids). ${ }^{27,28}$ Such voids provide bypasses through the MMMs that reduce the separation efficiency and compromise performance. ${ }^{29}$ In the present study, the shaping of MMMs requires the synthesis of MIL-96(Al) particles at the nanoscale. Therefore, the synthesis of MIL-96(Al) crystals of different morphology and diameter was achieved in water or water/DMF solvents. Two sets of nanoparticles of MIL-96(Al) with a monodisperse size distribution (200 and $70 \mathrm{~nm}$ in diameter) were then selected as inorganic fillers for the processing of MMMs. The glassy and high free-volume polyimide 6FDA-DAM was selected owing to its high $\mathrm{CO}_{2}$ permeability, good mechanical properties and easy processability. Defect-free membranes were prepared that consist of 
homogeneously distributed MIL-96(Al) particles in the polymer matrix with adequate interfacial properties. Their gas sorption properties were fully characterized, showing very encouraging performances for post-combustion $\mathrm{CO}_{2} / \mathrm{N}_{2}$ separation. Such results are very promising in the field of MMMs for $\mathrm{CO}_{2}$ capture.

\section{- EXPERIMENTAL SECTION.}

\section{Microwave Synthesis of MIL-96(AI) hexagonal platelets (MIL-96(Al)-HP). MIL-96(Al)} hexagonal platelets were synthesized by microwave assisted hydrothermal synthesis. Aluminum nitrate nonahydrate $(191.7 \mathrm{mg}, 0.511 \mathrm{mmol})$ and trimesic acid $(70.4 \mathrm{mg}, 0.335 \mathrm{mmol})$ were dissolved in distilled water $(45 \mathrm{~mL})$ under vigorous stirring. Acetic acid $(14 \mu \mathrm{L}, 0.25 \mathrm{mmol})$ was added, and the reaction mixture was stirred for $10 \mathrm{~min}$ at room temperature (RT) and then introduced into the microwave oven. After hydrothermal treatment at $200{ }^{\circ} \mathrm{C}(1600 \mathrm{~W})$ for $2 \mathrm{~min}$, the resulting mixture was cooled down with an ice bath and centrifuged at $14500 \mathrm{rpm}$ for $15 \mathrm{~min}$. The liquid fraction was discarded and the white product was washed twice with deionized water (30 $\mathrm{mL})$ and twice with EtOH $(30 \mathrm{~mL})$. After a final centrifugation, the powder was dried in air at RT.

Synthesis of MIL-96(AI) nanoparticles in water (MIL-96(AI)-NP1). A first solution was prepared by dissolving trimesic acid $(210 \mathrm{mg}, 1 \mathrm{mmol})$ in $70 \mathrm{~mL}$ of deionized water under reflux conditions. A second solution was obtained by dissolving aluminum nitrate nonahydrate (570 $\mathrm{mg}$, $1.5 \mathrm{mmol}$ ) in $65 \mathrm{~mL}$ of deionized water. Both solutions were heated separately under reflux and then the $\mathrm{Al}$ solution was poured into the ligand solution. Reflux was kept for about 3 hours and the white powder was cooled down in an ice bath before centrifugation at $14500 \mathrm{rpm}$ for $15 \mathrm{~min}$. The solid was washed twice with deionized water $(30 \mathrm{~mL})$ and twice with EtOH $(30 \mathrm{~mL})$. Pure MIL96(Al) nanoparticles were finally obtained and dried in air at RT.

\section{Synthesis of MIL-96(AI) nanoparticles in water/DMF (MIL-96(AI)-NP2 and NP3).} Aluminum nitrate nonahydrate $(4.5 \mathrm{~g}, 12 \mathrm{mmol})$ and trimesic acid $(2.52 \mathrm{~g}, 12 \mathrm{mmol})$ were dissolved in $300 \mathrm{~mL}$ of a $\mathrm{H}_{2} \mathrm{O} / \mathrm{DMF}$ (v:v =1/1) mixture. Acetic acid $(1.68 \mathrm{~mL}, 30 \mathrm{mmol})$ was added and the mixture was heated under reflux for 16 hours. The resulting white mixture was centrifuged at 14500 
rpm for $15 \mathrm{~min}$, and then washed once with deionized water $(30 \mathrm{~mL})$, one more time with a $\mathrm{H}_{2} \mathrm{O} / \mathrm{EtOH}(1 / 1)$ mixture $(30 \mathrm{~mL})$ and finally with EtOH $(30 \mathrm{~mL})$. The obtained white powder was dried at RT and pure MIL-96(Al) NP (i. e. MIL-96(Al)-NP2) of $200 \mathrm{~nm}$ in diameter were obtained, with no traces of trimesic acid, nitrates or DMF. MIL-96(Al) NP of $70 \mathrm{~nm}$ in diameter (i. e. MIL96(Al)-NP3) were also prepared at the gram scale by using the same reactants but with concentrations two times lower.

Preparation of MIL-96(Al)-6FDA-DAM MMMs. A high free-volume 6FDA-DAM copolymer supplied by Akron was used. 6FDA-DAM was degassed at $453 \mathrm{~K}$ under vacuum overnight. A polymer solution was prepared by dissolving $0.4 \mathrm{~g}$ of polymer in $3 \mathrm{~mL}$ of tetrahydrofuran (THF). MIL-96(Al) crystals (NP2 and NP3) were dispersed in $1.5 \mathrm{~mL}$ of THF by ultrasonication and stirring for $30 \mathrm{~min}$. Then, $10 \%$ of the volume of the polymer solution was added to the MOF suspension followed by further stirring for $2 \mathrm{~h}$ (priming). The remaining volume of polymer solution was added to the MOF suspension and stirred overnight. This slurry solution was poured on the glass plate and casted by Doctor Blade technique tuning the thickness to about $80 \mu \mathrm{m}$. The solvent/(filler + polymer) weight ratio was kept constant (90/10) for all MMMs. MMMs were prepared with a MOF loading of 25 wt. $\%$. Then, membranes were covered with a top-drilled box, and dried overnight under THF-saturated atmosphere. Finally, they were peeled off and heat treated at $433 \mathrm{~K}$ for $24 \mathrm{~h}$ under vacuum.

\section{— RESULTS AND DISCUSSION}

Synthesis of MIL-96(Al) and structure resolution by combining single-crystal X-ray Diffraction, solid-state NMR and DFT calculations.

The microporous MIL-96(Al) (see Figures 1 and 2) was first reported using hydrothermal conditions from a mixture of trimesic acid (BTC) and aluminum nitrate. ${ }^{15}$ However these conditions have to be strictly controlled since two larger-pore Al trimesates, MIL-100(Al) and MIL-110(Al), among others, can be produced in the same reaction system. ${ }^{19,30,31,32,33}$ Indeed, it was reported that MIL-100(Al) is the kinetic phase of this system and forms at short reaction times and low $\mathrm{pH}$, 
whereas MIL-96(Al) is mainly obtained for longer reaction times following a crystallizationhydrolysis-recrystallization process. ${ }^{31}$ In addition, this route involves the use of HF and tetraethoxysilane. ${ }^{15}$ We also failed to reproduce single crystals of MIL-96(Al) of a suitable size following this method. Thus, we developed first an alternative easier and friendlier hydrothermal route by using a mixture of trimesic acid and aluminum nitrate in water at $180^{\circ} \mathrm{C}$ (rather than $210^{\circ} \mathrm{C}$ ) which led to hexagonal rods (MIL-96(Al)-HR) up to $20 \mu \mathrm{m}$ long and $5 \mu \mathrm{m}$ wide. (See Figure 1(a) and Figure S1 of SI).

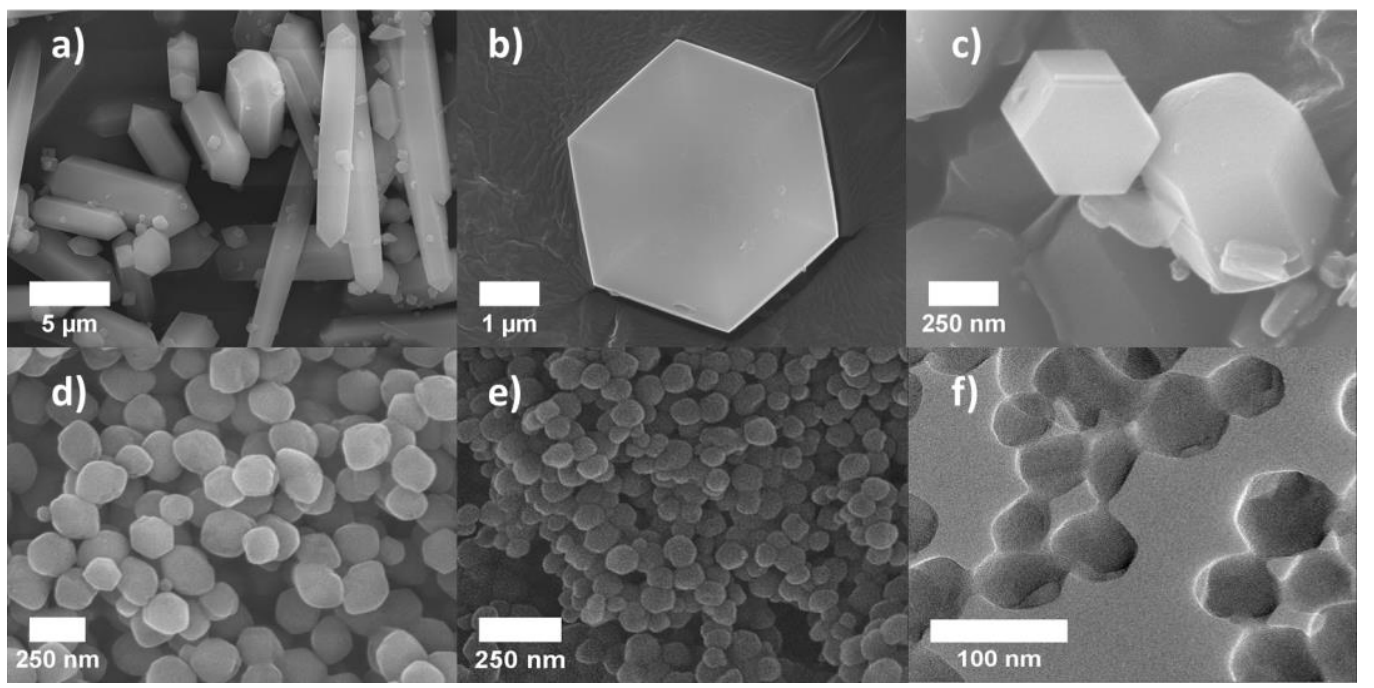

Figure 1. SEM images of the different MIL-96(Al) particles: a) Hexagonal rods (HR), b) hexagonal platelets (HP); c) nanoparticles from reflux in water (NP1); d) nanoparticles from reflux in $\mathrm{H}_{2} \mathrm{O} / \mathrm{DMF}$ (NP2); e) SEM image of NP3 and f) TEM-bright field image of NP3.

The structure of MIL-96 (Al), initially described by Loiseau et al., ${ }^{15}$ presents four non-equivalent aluminum sites. The fourth aluminum labelled as Al4, exhibits very short and unrealistic Al-O distances which suggests a crystallographic disorder of the initial structure. Therefore, in the present article, this structure has been reinvestigated using first microfocused beam synchrotron-based single crystal X-ray diffraction (see SI for details). As previously described, the structure results from the assembly of two distinct building units (see Figures 2(a) and (b)). The first one is an oxocentered trimer $\{\mathrm{Al}(1)\}$ of crystallographically equivalent $\mathrm{Al}(1)$ octahedra which are coordinated to bridging trimesate and $\mu_{3}-\mathrm{O}$ ligands (see Figure 2(c). The second building unit consists of a 2D hexagonal network containing 18-membered rings built by sinusoidal chains of aluminum octahedra. These chains contain two non-equivalent types of aluminum, $\mathrm{Al}(2)$ and $\mathrm{Al}(3)$, which are coordinated to four and two trimesate oxygen atoms and two and three bridging $\mu_{2}$-hydroxo 
moieties (Figures 2(d) and (e) respectively. The hexagonal 18-membered rings are interconnected each other by another type of trimer. This 'unusual' $\{\mathrm{Al}(3)\}$ trimer, seldom observed so far in the field of MOFs, is composed of three $\mathrm{Al}(3)$ octahedra that are corner-linked by $\mu_{2}-\mathrm{OH}$ groups. The connection of the $\{\mathrm{Al}(1)\}$ trimer with the $2 \mathrm{D}$ network of $\mathrm{Al}(2)$ and $\mathrm{Al}(3)$ octahedra through the trimesate ligands results in a 3D framework with a complex arrangement of three types of cavities (see Figure S2 and Table S1 of SI).

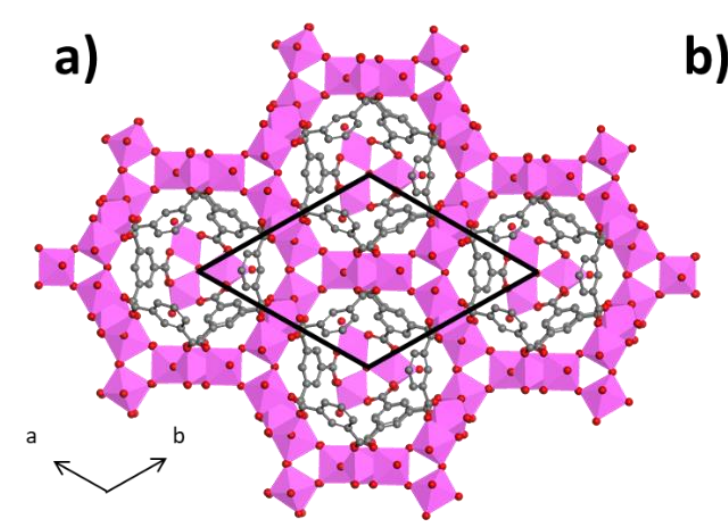

c)

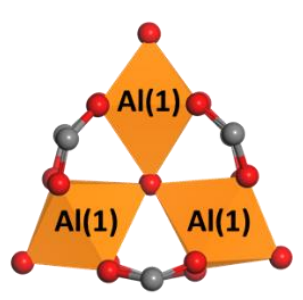

b)

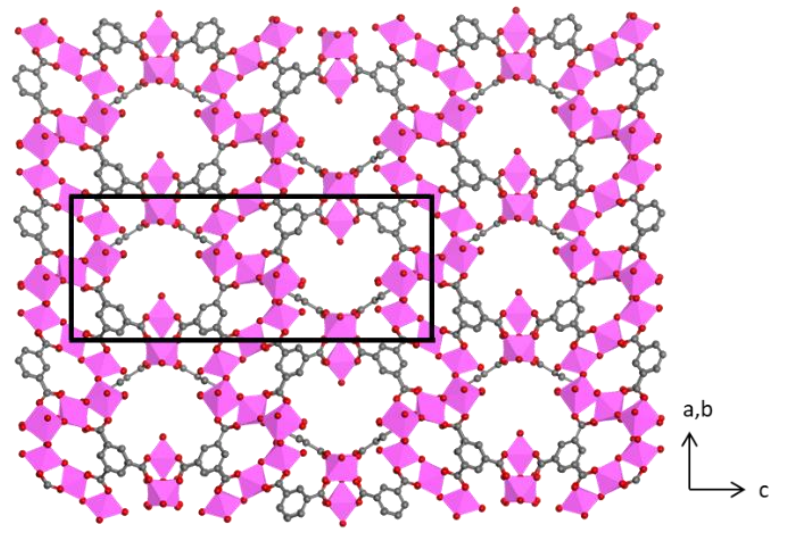

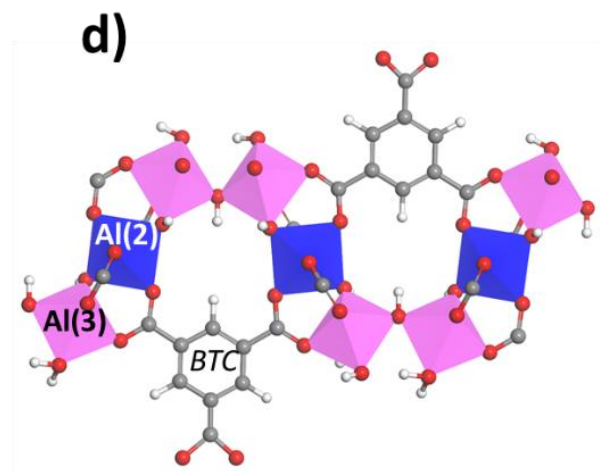

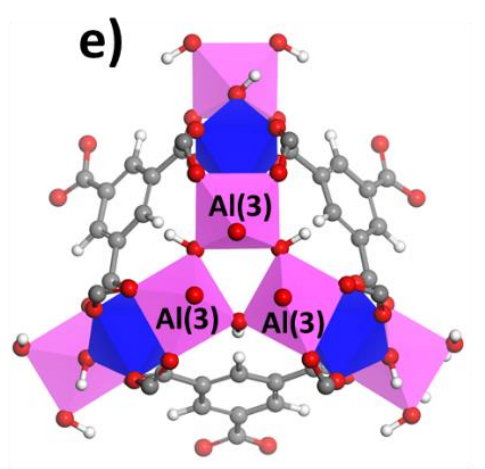

Figure 2. (a) Framework of MIL-96(Al) along c axis; (b) Framework of MIL-96(Al) along a axis; (c-e) Structural description of the different inorganic sub-units of MIL-96(Al): (c) $\{\mathrm{Al}(1)\}$ trimer (d) Sinusoidal chain composed of two $\mathrm{Al}(3)$ bridged by $\mathrm{Al}(2) ;(\mathrm{e})\{\mathrm{Al}(3)\}$ trimer.

The microporosity of MIL-96(Al) consists of one spherical cage A with a cavity-free diameter of about $11 \AA$, an elongated cavity B with dimensions of $9.5 \times 12.6 \times 11.3 \AA$ and a narrow cavity C with dimensions of $3.6 \times 4.5 \AA$. The complex packing and connectivity between cavities as described in Figure S3 shows that cavities of the same type (A-A, B-B or C-C) are not connected. According to the size of the windows between two cavities that could be estimated from the van der Waals radius of oxygen atoms, windows between cavity $\mathrm{B}$ and cavity $\mathrm{C}$ are relatively narrow $(\approx 4.5$ $\AA$ x $3.6 \AA$ ) and hence accessible to $\mathrm{H}_{2}, \mathrm{CO}_{2}$ and to a lesser extent to $\mathrm{N}_{2}$ (Figure S4) which is crucial for high $\mathrm{CO}_{2} / \mathrm{N}_{2}$ selectivity. On the other hand, no connection between cavity $\mathrm{A}$ and cavities $\mathrm{B} / \mathrm{C}$ is 
possible which means that cavity A is isolated in the structure. Consequently, MIL-96(Al) presents a two-dimensional (2D) pore structure that consists of "zig- zag" channels between cavities B and C (Figure S3). The nitrogen adsorption isotherm of MIL-96(Al) at $77 \mathrm{~K}$ (Figure S5(a)) is of type I(a) from the IUPAC classification which is characteristic of a microporous adsorbent. A BET area of $\sim 600 \pm 10 \mathrm{~m}^{2} \cdot \mathrm{g}^{-1}$ and a pore volume of $0.23 \mathrm{~cm}^{3} \cdot \mathrm{g}^{-1}$ are obtained. In addition, the semi-logarithmic plot of the $\mathrm{N}_{2}$ isotherm is consistent with a narrow pore size distribution (Figure S5(b)).

In contrast to the previously reported structure,${ }^{15}$ no electronic residue could be attributed to a fourth octahedral aluminum site. This observation contradicts the initial set of ${ }^{27} \mathrm{Al}$ MAS NMR spectroscopy data which evidenced the presence of four ${ }^{27} \mathrm{Al} \mathrm{NMR}$ signals in MIL-96(Al). ${ }^{15}$ In order to explain such a deviation, this phase was thoroughly investigated by combining solid state NMR, thermogravimetric analysis (TGA), and density functional theory (DFT) calculations. First, the ${ }^{27} \mathrm{Al}$ MQMAS NMR spectrum (see Figure S6(a) of SI) of the hydrated MIL-96(Al) shows only three ${ }^{27} \mathrm{Al}$ signals at $\delta_{\text {iso }}$ of 3.2, 3.4 and $6.2 \mathrm{ppm}$, which, according to the deconvolution of the ${ }^{27} \mathrm{Al}$ MAS NMR spectrum (Figure 3(a)) have respective relative intensity close to 1:1:2 (see Table S2 for the NMR parameters). This is consistent with the presence in the crystal structure of three $\mathrm{Al}$ sites with different multiplicity (the multiplicity of $\mathrm{Al}(1)$ and $\mathrm{Al}(2)$ is 6 while that of $\mathrm{Al}(3)$ is 12 ), and indicates that $\mathrm{Al}(3)$ corresponds to the line at $6.2 \mathrm{ppm}$. $\mathrm{Al}(1)$ and $\mathrm{Al}(2)$ can be distinguished based on the ${ }^{27} \mathrm{Al}-{ }^{27} \mathrm{Al}$ double-quantum single-quantum (DQ-SQ) NMR spectrum, in which close spatial proximities between ${ }^{27} \mathrm{Al}$ nuclei are observed. This spectrum (Figure 3(c)) shows a pair of crosspeak between $\mathrm{Al}(3)$ and the line at $3.4 \mathrm{ppm}$, which is thus assigned to the connected $\mathrm{Al}(2)$ center. $\mathrm{A}$ strong diagonal peak for the $\mathrm{Al}(3)$ resonance is observed, in agreement with the presence of connected $\mathrm{Al}(3)$ octahedra. For $\mathrm{Al}(1)$, only a diagonal peak is detected in agreement with the presence of isolated $\mathrm{Al}(1)$ sites. Upon dehydration of the MIL-96(Al) at $423 \mathrm{~K}$, removal of water molecules entrapped in the porosity of MIL-96(Al) takes place without altering the crystallographic structure of MIL-96(Al) as shown both by TGA and temperature-dependent Powder X-ray Diffraction (PXRD) (see Figures S7 and S8). However, the dehydration provokes a modification of the local environment of $\mathrm{Al}^{3+}$ cations since a fourth ${ }^{27} \mathrm{Al}$ resonance (labelled $\mathrm{Al}\left(3^{\prime}\right)$ ) is detected in 
a)
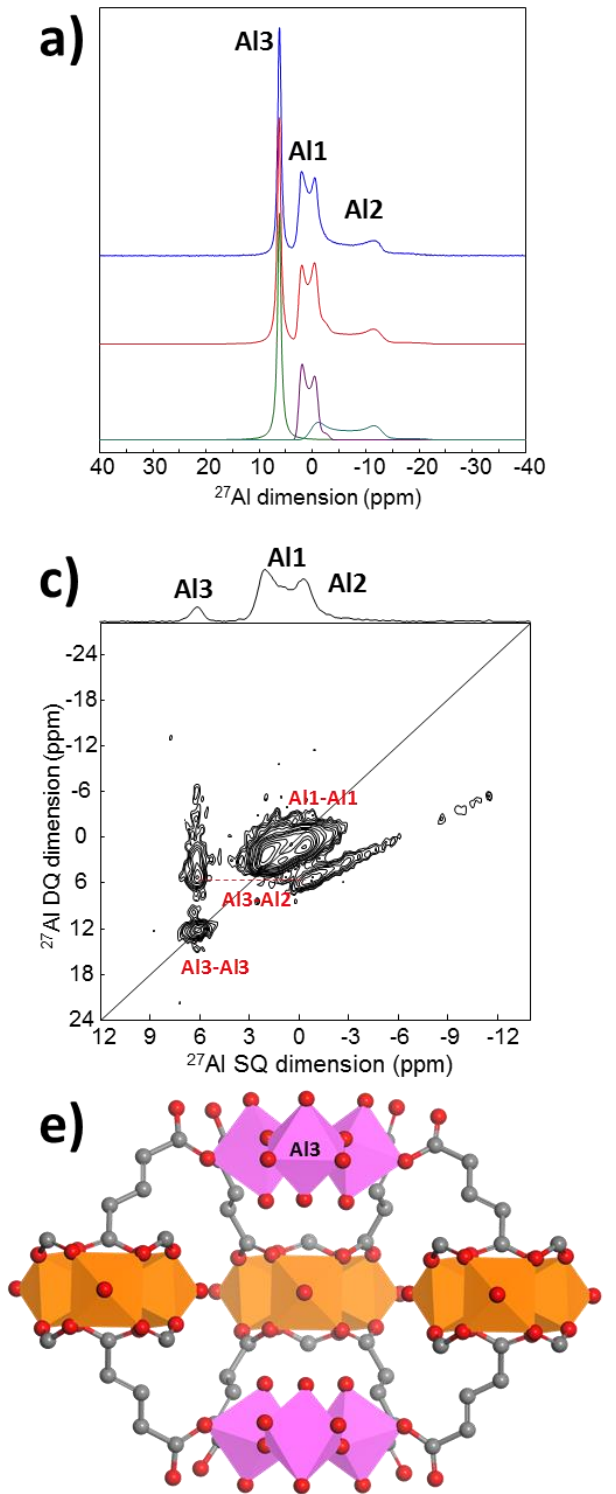

Dehydrated MIL-96(Al)

b)
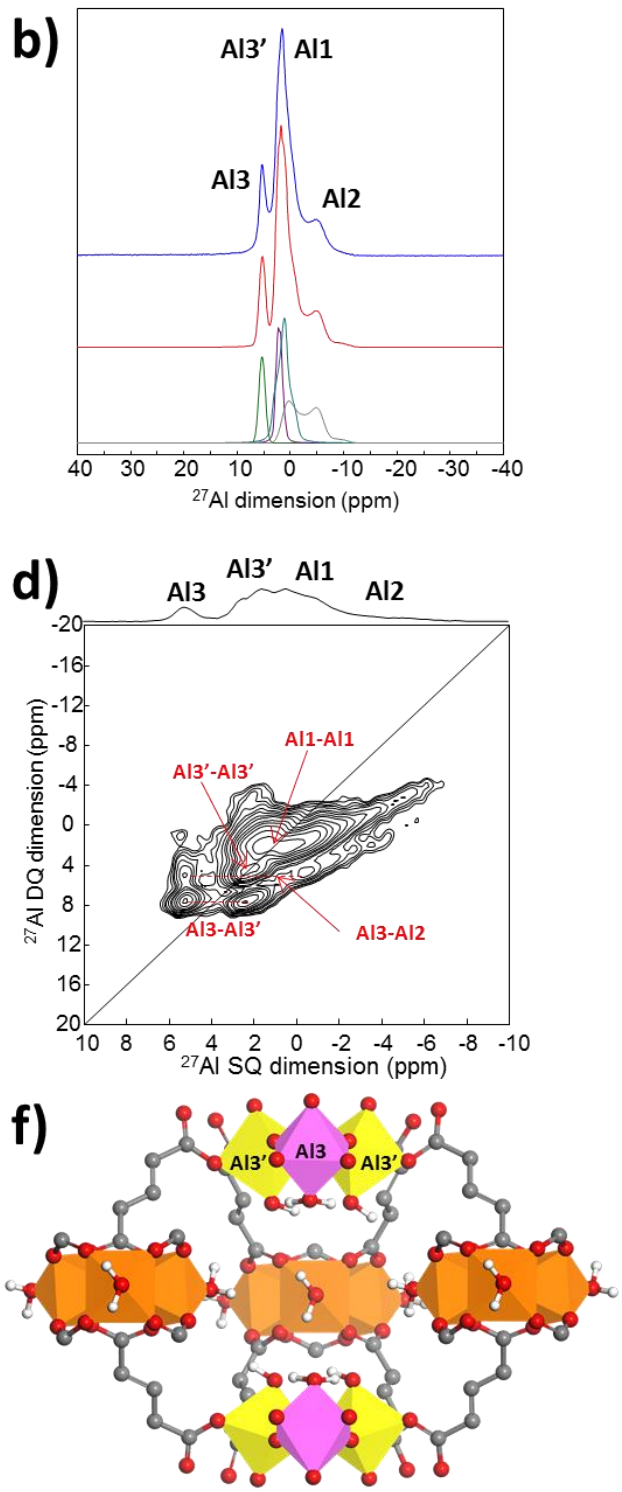

Figure 3. Experimental (Exp.) and deconvoluted (Dec.) ${ }^{27} \mathrm{Al}$ MAS NMR spectra of MIL-96(Al) (a) hydrated, (b) dehydrated (i.e. previously dried overnight at $423 \mathrm{~K}$ ). The individual contributions are shown below. ${ }^{27} \mathrm{Al}-{ }^{27} \mathrm{Al} \mathrm{DQ}-\mathrm{SQ}$ correlation NMR spectra of (c) hydrated MIL-96(Al) and (d) dehydrated MIL-96(Al). The diagonal has a slope of 2. For (c) the auto-correlations $\mathrm{Al}(3)-\mathrm{Al}(3)$ and $\mathrm{Al}(1)-\mathrm{Al}(1)$ are the diagonal peaks while the cross-correlation $\mathrm{Al}(3)-\mathrm{Al}(2)$ is indicated by the dashed line. For (d) the auto-correlations $\mathrm{Al}\left(3^{\prime}\right)-\mathrm{Al}\left(3^{\prime}\right)$ and $\mathrm{Al}(1)-\mathrm{Al}(1)$ are the diagonal peaks while the cross-correlation $\mathrm{Al}(3)-\mathrm{Al}\left(3^{\prime}\right)$ and $\mathrm{Al}(3)-\mathrm{Al}(2)$ are indicated by the dashed lines. (e,f) $\mathrm{A}$ view of the $\{\mathrm{Al}(1)\}$ and $\{\mathrm{Al}(3)\}$ trimers sub-units of (e) hydrated MIL-96(Al) and (f) dehydrated MIL-96(Al).

Moreover, deconvolution of the ${ }^{27} \mathrm{Al}$ MAS NMR spectrum indicates that the four signals at $\delta_{\text {iso }}=$ 3.0, 3.1, 3.4 and $5.3 \mathrm{ppm}$ have respective relative intensity of 1.3:1:1:0.66 (Figure 3(b) and Table $\mathrm{S} 2)$, and thus are assigned to $\mathrm{Al}\left(3^{\prime}\right), \mathrm{Al}(2), \mathrm{Al}(1)$ and $\mathrm{Al}(3)$, respectively. Note that the ${ }^{27} \mathrm{Al} \mathrm{NMR}$ spectrum reported previously by Loiseau et $\mathrm{al}^{15}$ was similar to our spectrum with the presence of 4 signals (one was assigned to the fourth aluminum site not present in our new model). However, the 
relative intensity of the signals is different, suggesting that the corresponding NMR spectrum was initially collected on a partially hydrated sample. This observation points out the importance of accurately controlling the hydration state of hydrophilic MOFs prior to NMR measurements. In the ${ }^{27} \mathrm{Al}-{ }^{27} \mathrm{Al} \mathrm{DQ}-\mathrm{SQ}$ NMR spectrum, the $\mathrm{Al}(3)$ resonance is no longer associated with a diagonal peak but strongly correlates with the signal of $\mathrm{Al}\left(3^{\prime}\right)$. All these results are consistent with the presence of one $\mathrm{Al}(3)$ and two equivalent $\mathrm{Al}(3)$ ' sites in each $\{\mathrm{Al}(3)\}$ trimer of the dehydrated MIL-96(Al).

According to the single crystal X-ray structure and TGA analysis (Figure S7), MIL-96(Al) with the chemical formula $\mathrm{Al}_{12} \mathrm{O}(\mathrm{OH})_{16}\left(\mathrm{H}_{2} \mathrm{O}\right)_{5}[\mathrm{BTC}]_{6} \cdot \mathrm{nH}_{2} \mathrm{O}(\mathrm{n}=29)$ contains one $\{\mathrm{Al}(1)\}$ trimer for two $\{\mathrm{Al}(3)\}$ trimers. The oxo-core of this formula unit is composed of one $\mu_{3}-\mathrm{O}$, twelve $\mu_{2}-\mathrm{OH}$ and nine terminal oxygen ligands $\left(\mathrm{OH}\right.$ or $\left.\mathrm{H}_{2} \mathrm{O}\right)$. For electroneutrality purposes, these terminal groups correspond to four hydroxyl groups and five water molecules that are linked either to $\mathrm{Al}(1)$ or $\mathrm{Al}(3) / \mathrm{Al}\left(3^{\prime}\right)$ sites. Indeed, $\mathrm{Al}(2)$ cations are not coordinated to any terminal oxygen ligands. Since $\mathrm{Al}(1)$ centers are chemically equivalent in the dehydrated MIL-96(Al), they are coordinated to one type of terminal oxygen ligand $\left(\mathrm{OH}\right.$ or $\left.\mathrm{H}_{2} \mathrm{O}\right)$ while the non-equivalent $\mathrm{Al}(3)$ and $\mathrm{Al}\left(3^{\prime}\right)$ centers are presumably not linked to the same terminal oxygen groups. Taking into account the total number of terminal $\mathrm{OH}$ and $\mathrm{H}_{2} \mathrm{O}$ and the symmetry of the $\left.\mathrm{Al}(1)\right\}$ and $\{\mathrm{Al}(3)\}$ trimers, the only possible configuration is that both $\mathrm{Al}(1)$ and $\mathrm{Al}(3)$ centers are coordinated to terminal water molecules while $\mathrm{Al}(3)^{\prime}$ is covalently attached to a hydroxyl group. However, a statistical distribution of hydroxyl groups and water molecules over the terminal oxygen ligands of $\mathrm{Al}(3) / \mathrm{Al}\left(3^{\prime}\right)$ cannot be ruled out.

The structural model of the anhydrous MIL-96(Al) thus proposed was geometry optimized at the DFT-level (Figure S29). These calculations included the relaxation of the atomic positions of the MOF framework while the unit cell parameters were fixed at the values determined experimentally. The same procedure was applied to the hydrated form of MIL-96(Al) starting with the experimental structural model containing a concentration of free water present in the pores as determined by TGA (58 $\mathrm{H}_{2} \mathrm{O} /$ u.c.) (Figure S7). In this case, the positions of both the atoms of the MOF framework and the free water molecules were relaxed. The so-obtained structural models were further validated by a good agreement between the first-principle calculated ${ }^{27} \mathrm{Al}$ NMR parameters including 
isotropic chemical shifts as well as quadrupolar coupling constants/asymmetric constants and the corresponding experimental data (see Table S2). The DFT predicted structure for the hydrated form reveals that the free water molecules make strong hydrogen bonds with the framework ( $\mathrm{Al}$ centres or hydroxy groups, trimesate ligands, or coordinating water molecules) (see Fig. 4(a)).
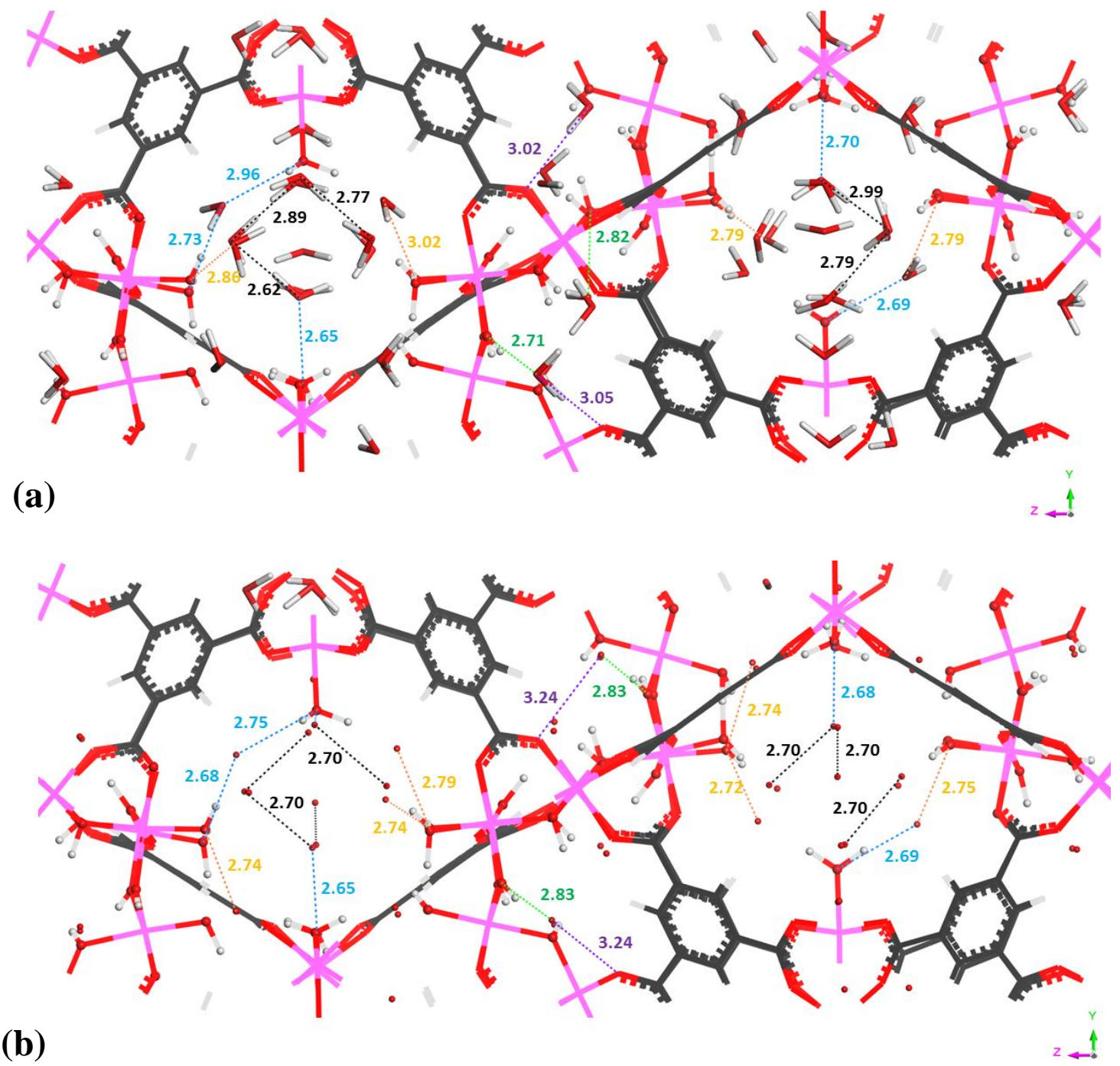

Figure 4. Crystal structure of the MIL-96(Al) in its hydrated form. (a) DFT optimized model, (b) refined experimental model. (Gray, carbon; white, hydrogen; red, oxygen; pink, aluminum) Interatomic distances are in Angströms. The dashed lines represent a selection of representative interactions between the water molecules themselves (black), between water and water coordinated to framework (blue), between water and hydroxyl group coordinated to framework (orange), between the water and the $\mu_{2}-\mathrm{OH}$ functions (green) and between the water and the carboxylate group Oc (purple). Hydrogen bonds are involved between free water molecules and coordinative water molecules at the $\mathrm{Al}(1)$ sites (blue). Water molecules located near to the $\mathrm{Al}(2)$ sites are arranged in such a way to form hydrogen bonds with the framework between 1) their oxygen atoms Ow and the proton of the $\mu_{2}(\mathrm{OH})$ functions (orange) and 2) their protons $\mathrm{Hw}$ and the oxygen atoms of the carboxylate group (purple). In addition, the water molecules located near to $\mathrm{Al}(3)$ sites make hydrogen bonds with framework between 1) their protons $\mathrm{Hw}$ and the oxygen atoms of either the hydroxyl group (orange) or water coordinated to $\mathrm{Al}\left(3^{\prime}\right)$ and/or $\mathrm{Al}(3)$ sites (blue) and 2) their oxygen atoms $\mathrm{Ow}$ and the proton of the nearby water molecules (black).

The characteristic distances between two water/host oxygen atoms range from 2.6 and $3.05 \AA$, which fit well with those evidenced from the experimentally-refined structure. The confined water molecules form a relatively strong hydrogen bond network with corresponding Ow-Ow distances 
$(2.77 \AA)$ as short as those usually observed for water in the bulk state. This $\mathrm{H}_{2} \mathrm{O}$ network is extended throughout the porosity by hydrogen bonded water molecules between cavities B and C as shown in Figure S9. Finally, a theoretical accessible surface area of $700 \mathrm{~m}^{2} \cdot \mathrm{g}^{-1}$ was evaluated, in fair agreement with the BET area of $600 \mathrm{~m}^{2} \cdot \mathrm{g}^{-1}$ (see vide supra), thereby providing additional evidence of the reliability of the so-built structural models.

\section{Control of the particle size and morphology: synthesis of nanoparticles of MIL-96(Al).}

The characteristic morphology of MIL-96(Al) crystals, as initially described, is that of a truncated hexagonal bipyramid. ${ }^{15}$ Several solvo- and hydrothermal routes led to MIL-96(Al) crystals with such a morphology but with different diameters (ranging from $0.8 \mu \mathrm{m}$ to $40 \mu \mathrm{m}$ ) using $\mathrm{Me}_{3} \mathrm{BTC}$ or trimesic acid. ${ }^{19,31,34,35}$. Finally, nanoparticles of MIL-96(Al) (from $160 \mathrm{~nm}$ to $55 \mathrm{~nm}$ ) were recently reported by Knebel et al. ${ }^{18}$ following a solvothermal synthesis in a mixture of $\mathrm{H}_{2} \mathrm{O} / \mathrm{DMF}$ solvents We report here several synthesis routes yielding to pure MIL-96(Al) with various morphology and size, down to the nanoscale as required for further MMM preparation. Microwave (MW)-assisted synthesis is known to reduce the size and the polydispersity of the particles due to fast and homogeneous heating favoring the nucleation process. ${ }^{36}$ In contrast to the synthesis conditions of MIL-96(Al)-HR, the initial aqueous solution of both aluminum and ligand precursors was diluted and an amount of acetic acid was added. A 2 minute microwave assisted hydrothermal synthesis performed at $473 \mathrm{~K}$ led to pure MIL-96(Al) hexagonal platelets (i. e. MIL-96(Al)-HP), with a diameter of a few micrometers and an average thickness of a few $100 \mathrm{~nm}$ as determined by SEM (see Figure 1(b)). This process shows that MIL-96(Al) can be isolated even at short reaction times as a pure phase without the presence of MIL-100(Al) or MIL-110(Al). Noteworthy, PXRD of both MIL-96(Al)-HR and -HP (Figure 5(a)) show changes in relative intensity of several Bragg peaks indicating a preferential orientation of such crystals with a shape anisotropy. This phenomenon is noticeable by comparing PXRD recorded with reflection (Bragg-Brentano method) and transmission (Debye-Sherrer method) geometry for HR (Figure S10) and HP (Figure S11). Hexagonal rods present a strong (010) reflection while the intensity of $(00 l)$ peaks is reduced, and 
(002) almost extinct. On the other hand, $(00 \mathrm{l})$ and $(\mathrm{Okl})$ reflections are much more intense in crystals with hexagonal platelet morphologies. Therefore, $(0 \mathrm{kO})$ (and thus $(\mathrm{hOO})$ ) reflections can be assigned to the long facets of hexagonal rods while $(0 \mathrm{kl})$ (and by symmetry $(\mathrm{hol})$ ) reflections correspond to the trigonal facets present on top of the hexagonal platelets (see Figure $5(b, c)$ ), in agreement with that previously reported for MIL-96(Al) crystals of similar morphology. ${ }^{18}$ Since acetic acid may act as a modulator as reported for several polycarboxylate based MOFs, ${ }^{37,38}$ it can be suggested that the presence of acetic acid impacts the nucleation and growth of MIL-96(Al) crystals and thus its morphology. However, even without this modulator, MIL-96(Al)-HP was also obtained along with MIL-100(Al) as a by-product. Such results emphasize that the formation of trimesate Al-based MOFs strongly depends on the speciation of molecular Al complexes in solution. The MW synthesis conditions were transferred to reflux synthesis in water. An aqueous diluted solution of Al salt, trimesic acid and acetic acid was heated at reflux $\left(\sim 100^{\circ} \mathrm{C}\right)$ for a variable period of time. After only 3 hours at reflux, the colorless solution becomes cloudy indicating the formation of MIL96(Al) nanoparticles (i. e. MIL-96(Al)-NP1) as shown by PXRD (Figure 5(a)). As observed by SEM (Figure 1(c)), hexagonal nanorods with a dimension up to $500 \mathrm{~nm}$ are formed by this route. This is, to our knowledge, the first synthesis producing pure MIL-96(Al) nanoparticles in water under reflux conditions, with however a quite low yield (150 mg, $Y=45 \%$ based on $\mathrm{Al})$. Increasing the concentration of the reactants failed to produce MIL-96(Al). The formation of MIL-96(Al)-NP1 under reflux in water is certainly mainly limited by the poor solubility of trimesic acid in pure water. Therefore, half of the volume of water was replaced by DMF to dissolve the entire $12 \mathrm{mmol}$ of trimesic acid. After 16h of reflux, nanoscale MIL-96(Al) crystals were obtained as indicated by the presence of broad Bragg peaks on the XRD pattern (i.e. MIL-96(Al) NP2, see Figure 5(a)). As shown by the structureless refinement (Figure S12) and SEM images (Figure 1(d) and Figure S13), MIL-96(Al) NP2 consists of pure nanocrystals with a diameter of $200 \pm 30 \mathrm{~nm}$. It is worth noting, that synthesis conditions without acetic acid led to MIL-96(Al) nanocrystals with a similar size and shape, however with a larger polydispersity in diameter (i. e. diameter of particles of $190 \pm 100 \mathrm{~nm}$, see Figure S14). Such results are consistent with those previously reported by Sindoro et al. ${ }^{34}$ 

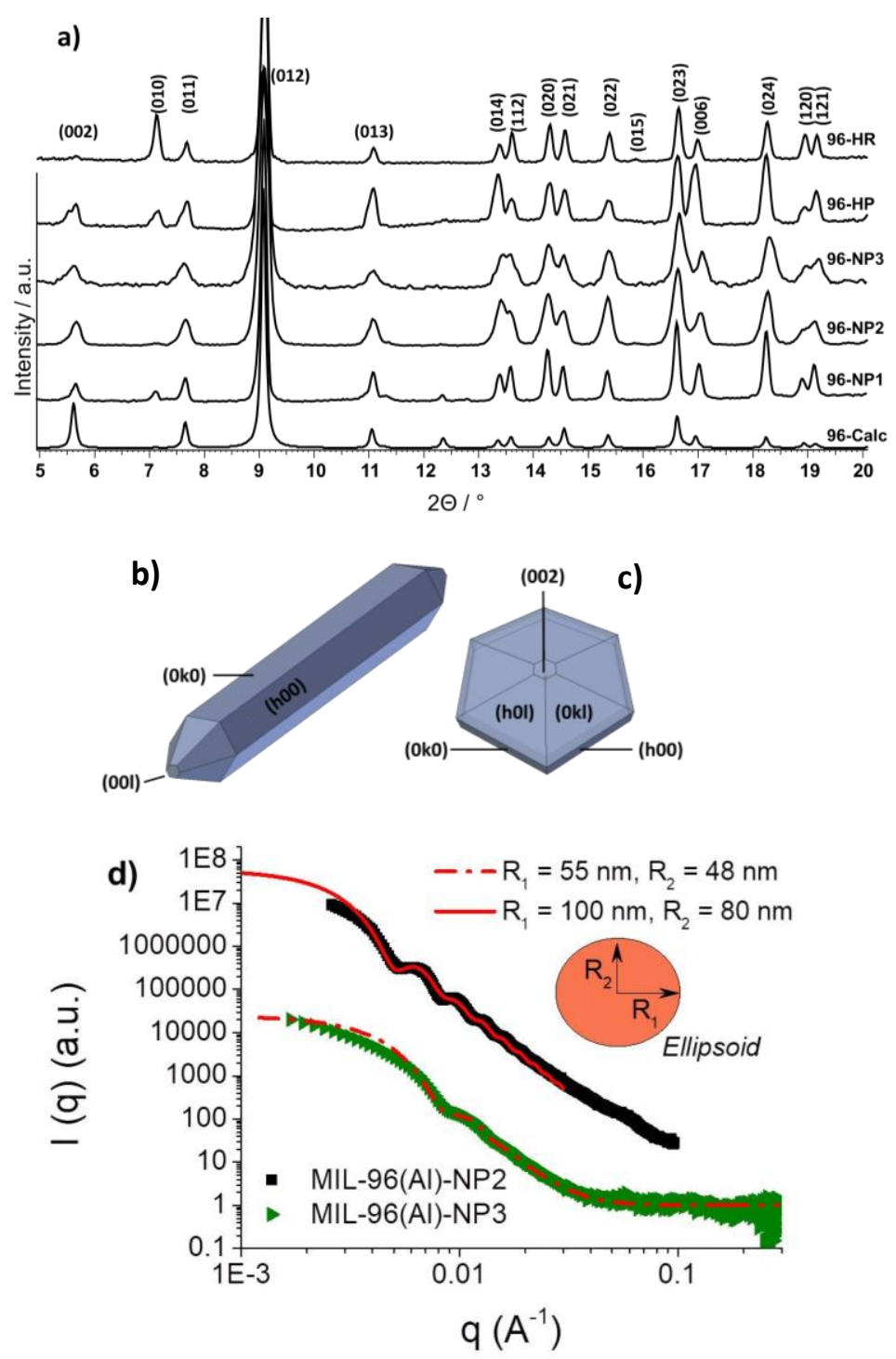

Figure 5. (a) PXRD of the different MIL-96(Al) particles. All patterns were normalized using the (012) reflection; (b, c) 3D representation of the crystal morphology and the ( $h k l$ ) planes of MIL-96(Al) b) Hexagonal rods and c) Hexagonal platelets. (d) SAXS curves of MIL-96(Al)-NP2 (black square) and MIL-96(Al)-NP3 (green triangle) solutions at $0.1 \mathrm{~g} / \mathrm{L}$ in water obtained at Alba and Soleil synchrotrons respectively. The red lines correspond to the best fit with the ellipsoid form factor with radius indicated in the inset and log-normal polydispersity. For the continuous line the polydispersity index (PD) are: $P D R_{1}=0.01$ and $P D R_{2}=0.15$ while for dash-dot line $\mathrm{PD}_{1}=0.1$ and $\mathrm{PD} \mathrm{R}_{2}=0.2$.

Following this synthetic route, a large amount of MIL-96(Al)-NP2 (e.g. $13 \mathrm{~g}$ in one batch with $Y=$ $96 \%$ based on $\mathrm{Al}$ ) was obtained. By decreasing by two folds the concentration of the reactants, smaller MIL-96(Al) NP were synthesized at the gram scale (similar yield than for NP2) as shown by PXRD (i.e. MIL-96(Al)-NP3, see Figure 5(a)). The structureless refinement (Figure S15) indicates MIL-96(Al) nanocrystals with an average diameter of $70 \mathrm{~nm}$, in agreement with the size distribution of $67 \pm 40 \mathrm{~nm}$ given by SEM and TEM images (Figures 1(e) and 1(f) and Figure S16 of SI). Nitrogen adsorption/desorption measurements $(77 \mathrm{~K})$ can be used to extract an 'external surface area' (i.e. without the porosity) and it would be expected that the smaller the particles, the higher the external surface area. The t-plot analysis was used which leads to values of 6,40 and $100 \mathrm{~m}^{2} / \mathrm{g}$ 
for the $-\mathrm{HR}$, -NP2 and -NP3 samples highlighting the increasing nanoparticular size of this series. (Figure S17). Dilute solutions of MIL-96(Al)-NP2 and MIL-96(Al)-NP3 in water were then studied by Small Angle X-Ray Scattering (SAXS). The SAXS curves (Figure 5(d)) are fairly well fitted on the whole q-range by the form factor of ellipsoids with rather low index of polydispersity. The dimensions extracted from fit $\left(\mathrm{R}_{1}=55 \mathrm{~nm}(\mathrm{PD}=0.1)\right.$ and $\mathrm{R}_{2}=48 \mathrm{~nm}(\mathrm{PD}=0.2)$ for MIL-96(Al)$\mathrm{NP} 3 ; \mathrm{R}_{1}=100 \mathrm{~nm}(\mathrm{PD}=0.01)$ and $\mathrm{R}_{2}=80 \mathrm{~nm}(\mathrm{PD}=0.15)$ for MIL-96(Al)-NP2) are in fair agreements with SEM observations. Noteworthy, no nanoparticle aggregation could be detected in the available q-range. Finally, dynamic light scattering (DLS) experiments with 1.25 wt\% MIL96(Al)-NP2 and -NP3 dispersed in pure tetrahydrofuran (THF) were performed to better characterize the colloidal stability. This solvent is currently used for dissolving 6FDA-DAM. DLS measurements (Figure S18) have shown only one population centered at $220 \mathrm{~nm}$ and $70 \mathrm{~nm}$ in diameter for MIL-96(Al)-NP2 and -NP3, respectively, indicating the absence of any aggregation even at such high concentrations $\left(1.25 \mathrm{wt} \% \approx 10 \mathrm{~g} . \mathrm{L}^{-1}\right)$ that are used for MMMs preparation. The colloidal solution is stable for at least 6 hours, which is of high interest for the preparation of MMMs since the drying process takes generally a few hours.

\section{In situ IR spectroscopy: characterization of adsorbed water, hydroxyl groups and $\mathrm{Al}^{3+}$ sites. $^{2}$}

FTIR spectra were recorded to study the stability and location of adsorbed water, characterize both the hydroxyl groups and $\mathrm{Al}$ sites of the MOF and finally probe the interactions between the MOF and different guest molecules. Detailed interpretations of the IR spectra are provided in the SI. The IR spectrum of the as-prepared hydrated MIL-96(Al)-HR sample (see Figure 6A, spectrum a) shows (i) the lack of free trimesic acid (evidenced by the absence of bands in the $1740-1710 \mathrm{~cm}^{-1}$ region), ${ }^{39,40}$ (ii) a significant amount of adsorbed water $\left(\mathrm{OH}\right.$ stretching band at $3388 \mathrm{~cm}^{-1}$ and combination band at $5215 \mathrm{~cm}^{-1}$, as well as a $\delta\left(\mathrm{D}_{2} \mathrm{O}\right)$ band at ca. $1205 \mathrm{~cm}^{-1}$ recorded with the deuterated sample) ${ }^{41}$ and (iii) the existence of some amount of "free"-OH groups that are not involved in H-bonding with adsorbed water (combination band around $4690 \mathrm{~cm}^{-1}$ and deformation band at $\left.1071 \mathrm{~cm}^{-1}\right) .^{41}$ The position of the latter IR bands indicates a very low acidity of (at least part 
of) the $\mathrm{OH}$ groups. Since the activation procedure for MOFs strongly impacts their sorption capacity, FTIR spectra of the MIL-96(Al)-HR sample were recorded after outgassing at increasing temperature. Water trapped in the MOF is mostly removed by outgassing at RT as evidenced by the strong decrease in intensity of the bands at 5215 and $3388 \mathrm{~cm}^{-1}$ (Figure 6(A), spectrum b). Only $\mathrm{H}_{2} \mathrm{O}$ coordinated to $\mathrm{Al}$ sites remains in the pores after evacuation at $323 \mathrm{~K}$, as shown by a weak $v(\mathrm{OH})$ band at $3415 \mathrm{~cm}^{-1}$, two weak combination bands at 5364 and $5288 \mathrm{~cm}^{-1}$ and, for the deuterated sample, two $\delta\left(\mathrm{D}_{2} \mathrm{O}\right)$ components at 1218 and $1209 \mathrm{~cm}^{-1}$ (Figure 6(B), right inset).
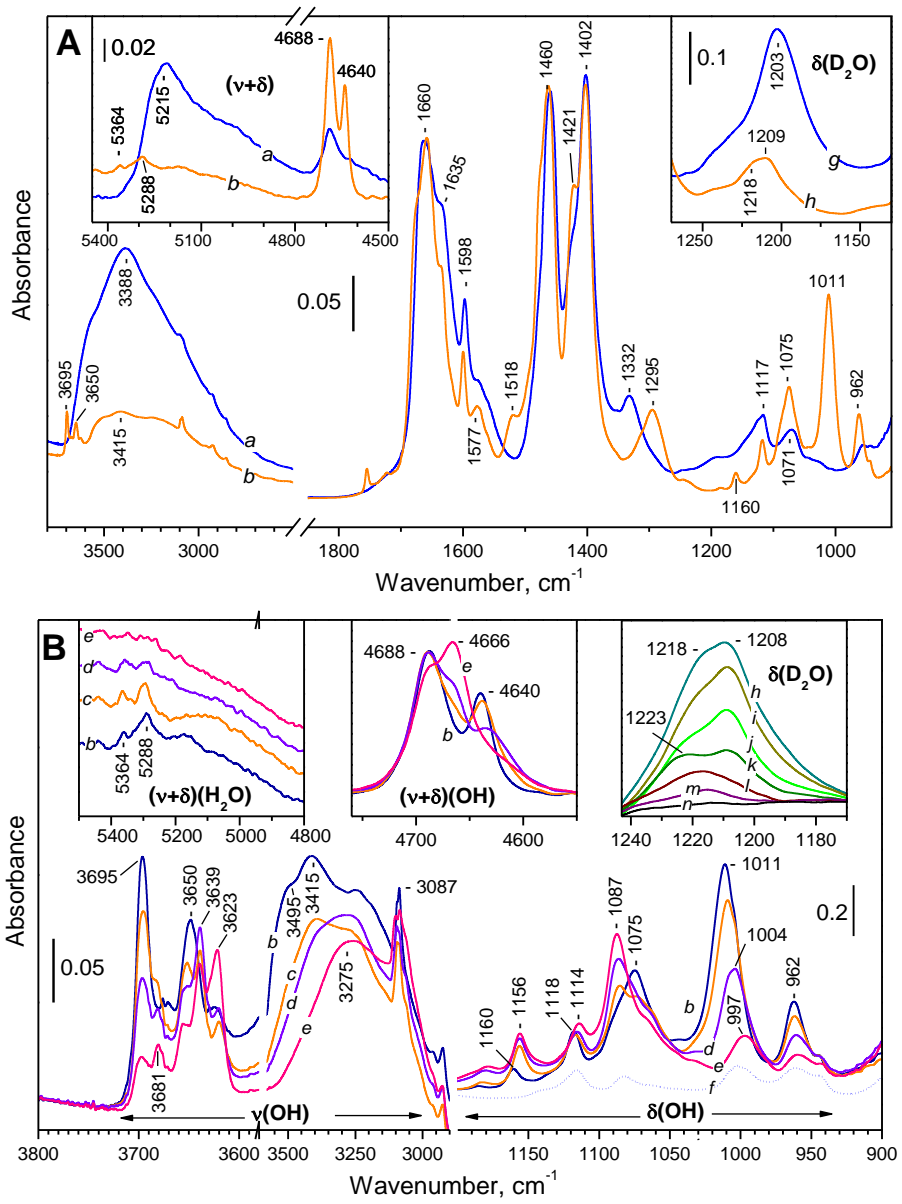

Figure 6. (A) IR spectra of MIL-96(Al)-HR: (a) as prepared sample and (b) after activation at RT. The left inset shows the spectrum in the $(v+\delta)$ region. The right inset shows the $\delta\left(\mathrm{D}_{2} \mathrm{O}\right)$ mode with a deuterated sample $(\mathrm{g})$ under $\mathrm{D}_{2} \mathrm{O}$ vapour and (h) after activation at RT. (B) IR spectra of MIL-96(Al)-HR in the $v(\mathrm{OH})$ and $\delta(\mathrm{OH})$ regions. Sample activated at (b) RT, (c) $373 \mathrm{~K}$, (d) $423 \mathrm{~K}$, (e) $473 \mathrm{~K}$ and (f) deuterated sample activated at $473 \mathrm{~K}$. The left and the middle insets show the spectra in the $(v+\delta)$ region of $\mathrm{H}_{2} \mathrm{O}$ and $\mathrm{OH}$ groups, respectively. The right inset shows the $\delta\left(\mathrm{D}_{2} \mathrm{O}\right)$ mode with a deuterated sample after activation at (h) RT, (i) $323 \mathrm{~K}$, (j) $348 \mathrm{~K}$, (k) $373 \mathrm{~K}$, (l) $398 \mathrm{~K}$, (m) $423 \mathrm{~K}$ and (n) $448 \mathrm{~K}$.

Further, the remaining water is removed after evacuation at 423-448 $\mathrm{K}$, in agreement with TGA results. The removal of the water leads to the appearance of "free" OH groups, as shown by two characteristic combination bands at 4688 and $4640 \mathrm{~cm}^{-1}$. In addition, a set of bands appears in the 
$v(\mathrm{OH})$ region, the assignment of which is more complicated due to the possible overlapping between $\mathrm{OH}$ vibrations of adsorbed water and free hydroxyl groups. ${ }^{41}$ For a correct assignment, partly deuterated samples were also investigated, thereby showing that all bands above $3600 \mathrm{~cm}^{-1}$ are due to $-\mathrm{OH}$ groups (see details in Figure S19 of SI). Therefore, the water $\mathrm{OH}$ modes appear below $3600 \mathrm{~cm}^{-1}$ which suggests that the two protons of residual adsorbed water are involved in $\mathrm{H}$ bonding with basic sites (presumably oxygen atoms of carboxylates) from the MOF structure.

Table 1. Vibrational bands of the hydroxyl groups of MIL-96(Al).

\begin{tabular}{|c|c|c|c|c|c|}
\hline Type of OH group & Notation & $v(\mathrm{OH})$ & $v(\mathrm{OD})$ & $\delta(\mathrm{OH})$ & $(v+\delta)(\mathrm{OH})$ \\
\hline Terminal & T1 & 3695 & 2724 & 962 & 4640 \\
\hline Terminal & T2 & 3681 & 2714 & 997 & - \\
\hline Bridging & B1 & 3650 & 2692 & 1011 & 4640 \\
\hline Bridging & B2 & 3639 & 2683 & 1075 & 4690 \\
\hline Bridging & B3 & 3623 & 2672 & 1087 & 4665 \\
\hline H-bonded in C cages & C & 3275 & 2448 & 1160 & - \\
\hline
\end{tabular}

The T-type species $\left(3695,3681 \mathrm{~cm}^{-1}\right)$ are assigned to structural terminal Al-OH hydroxyl while the B type species $\left(3650,3639,3623 \mathrm{~cm}^{-1}\right)$ can be attributed to bridging $\mu_{2}-\mathrm{OH}$ type groups. ${ }^{41}$ In addition, stable $\mathrm{H}$-bonded $\mathrm{OH}$ groups (C-type) were also detected by a band at $3275 \mathrm{~cm}^{-1}$. (Figure $6 \mathrm{~B}$, spectrum $\mathrm{d})$. The low $v(\mathrm{OH})$ frequency and the large band width indicate that these hydroxyls are also involved in strong H-bonding with basic sites from the walls and are likely confined in small pores (presumably cages $\mathrm{C}$ ). The bands of the terminal hydroxy develop after evacuation at RT as a result of the breaking of $\mathrm{OH} \cdots\left(\mathrm{H}_{2} \mathrm{O}\right)$ bonds which confirms the very weak acidity of these hydroxyls. Upon evacuation, the intensity of these bands decreases above $323 \mathrm{~K}$ pointing out some possible dehydroxylation process (for details see Figure S20). Bridging and C-type hydroxy groups are thermally stable. (see Figures S19-20 and the SI for more details). As shown by ${ }^{27} \mathrm{Al}$ NMR spectroscopy, a modification of the $\mathrm{Al}$ environment takes place at temperatures higher than $473 \mathrm{~K}$ : a ${ }^{27} \mathrm{Al}$ resonance centred around $35 \mathrm{ppm}$, corresponding to five-coordinated $\mathrm{Al}$ sites, appears (Figure S21). Finally, we studied samples activated at different temperatures and then subjected to water vapor and evacuated again at ambient temperature (Figure S22). Noteworthy, the reversibility of water adsorption/desorption and the excellent stability of MIL-96(Al) under water atmosphere or 
after thermal evacuation treatments was confirmed as the final spectra are essentially the same for samples evacuated up to $473 \mathrm{~K}$.

In order to probe the accessibility of the cavities of MIL-96(Al)-HR for gas sorption and to assess the Brønsted and Lewis acidity, adsorption of different probe molecules $\left(\mathrm{CO},{ }^{15} \mathrm{~N}_{2}\right.$ and $\left.\mathrm{CO}_{2}\right)$ was monitored by in situ FTIR spectroscopy. Before such experiments, MIL-96-(Al)-HR was evacuated at different temperatures. Details are described in the SI (Figures S23-26 of SI). While the pores of MIL-96(Al)-HR evacuated at RT are not accessible to $\mathrm{CO}$ or ${ }^{15} \mathrm{~N}_{2}$ at $\mathrm{T}=100 \mathrm{~K}$, a slight accessibility of the pores is observed after activation at $423 \mathrm{~K}$ that increases with the activation temperature (up to $573 \mathrm{~K}$ ). Upon $\mathrm{CO}$ adsorption, $\mathrm{OH}$ groups are detected through a carbonyl band at $2155-2152 \mathrm{~cm}^{-1} 42$ which develops with the pre-evacuation temperature up to $473 \mathrm{~K}$ (due to an increase in accessibility) and then decreases as a result of a possible dehydroxylation. Since two kinds of terminal $\mathrm{OH}$ groups (T1 and $\mathrm{T} 2$ ) differ only in the extent of $\mathrm{H}$-bonding to the framework, the formation of $\mathrm{OH} \cdots \mathrm{CO}$ adducts leads to a red shift of the two $\mathrm{OH}$ modes to one band at 3669 $\mathrm{cm}^{-1}$. This phenomenon indicates breaking of the pre-existing $\mathrm{H}$-bond and the shift value is consistent with the very weak acidity of the hydroxyls. The same phenomena are observed with the bridging hydroxyls. Some $\mathrm{Al}^{3+}$ coordinatively unsaturated sites (CUS) are also detected by $\mathrm{CO}$ or ${ }^{15} \mathrm{~N}_{2}$ (adsorbed at $100 \mathrm{~K}$ ) with a sample evacuated at temperatures as high as $423 \mathrm{~K}$. In contrast to the low-temperature experiments, adsorption of $\mathrm{CO}_{2}$ on a sample evacuated at RT leads to the formation of $\mathrm{Al}^{3+} \ldots \mathrm{OCO}\left(\mathrm{v}_{3}\left(\mathrm{CO}_{2}\right)\right.$ at $\left.2345 \mathrm{~cm}^{-1}\right)$ and $\mathrm{OH} \cdots \mathrm{OCO}$ adducts $\left(2338 \mathrm{~cm}^{-1}\right){ }^{43,44}$ The amount of the former species increases with the pre-evacuation temperature up to $473 \mathrm{~K}$ due to the removal of coordinated water while that of $\mathrm{OH} \cdots \mathrm{OCO}$ decreases.

\section{$\mathrm{CO}_{2} / \mathrm{N}_{2}$ gas sorption properties of MIL-96(Al)-NP2: single gas adsorption, $\mathrm{CO}_{2} / \mathrm{N}_{2}$ co-} adsorption and molecular simulations.

According to IR spectroscopy, MIL-96(Al) needs to be activated by outgassing the sample to around $423 \mathrm{~K}$ in order to remove all the free water or residual free trimesic acid. Single gas adsorption experiments were first performed on MIL-96(Al)-NP2 samples. Figure 7(a) shows the 
excess adsorption isotherms of pure single gas components $\mathrm{CO}_{2}$ and $\mathrm{N}_{2}$ obtained at $303 \mathrm{~K}$ for MIL96(Al)-NP2 activated at $423 \mathrm{~K}$ (see Fig. S27 for comparison with isotherms on MIL-96(Al)-HR). At 1 bar, the adsorption capacities are $3.2 \mathrm{mmol}_{\mathrm{g}} \mathrm{g}^{-1}$ for $\mathrm{CO}_{2}$ while much lower capacity of about 0.2 mmol.g $\mathrm{g}^{-1}$ for $\mathrm{N}_{2}$ is obtained. Grand Canonical Monte Carlo (GCMC) simulations were carried out at $303 \mathrm{~K}$ to predict the single component adsorption of $\mathrm{CO}_{2}$, and $\mathrm{N}_{2}$ (see SI for details). The experimental findings are qualitatively reproduced by the GCMC simulations (see Figure 7(a)), even though the calculated amounts adsorbed slightly overestimate the experimental data for the two gases. This discrepancy is likely due to the fact that GCMC simulations consider that both gases can adsorb in the entire porosity available in the solid. As explained above, the adsorption of gas in MIL-96(Al) might be restricted to cavities B and C since cavities A seem to be isolated in the framework. Interestingly, the experimental adsorption uptakes of $\mathrm{CO}_{2}$ at 1 bar $\left(\sim 3.2 \mathrm{mmol}^{-1}\right.$ at $303 \mathrm{~K})$ and 10 bar $\left(\sim 6.3 \mathrm{mmol.g}{ }^{-1}\right)$ are among the best performances for small pore MOFs previously reported for $\mathrm{CO}_{2}$ separation such as MIL-91(Ti) $\left(\sim 3.0 \mathrm{mmol} . \mathrm{g}^{-1}\right.$ at $1 \mathrm{bar} / 303 \mathrm{~K}$ and $\sim 4.5$ mmol.g ${ }^{-1}$ at $\left.10 \mathrm{bar} / 303 \mathrm{~K}\right),{ }^{45} \mathrm{MIL}-53(\mathrm{Al})-\mathrm{NH}_{2}\left(1.6 \mathrm{mmol} . \mathrm{g}^{-1}\right.$ at $283 \mathrm{~K}$ and $\left.1 \mathrm{bar}\right),{ }^{46} \mathrm{Sc}_{2}(\mathrm{BDC}-$ $\left.\mathrm{NO}_{2}\right)_{3}\left(1.1 \mathrm{mmol} . \mathrm{g}^{-1}\right.$ at $303 \mathrm{~K}$ and $\left.1 \mathrm{bar}\right),{ }^{47} \mathrm{UiO}-66(\mathrm{Zr})-2 \mathrm{COOH}\left(1.0 \mathrm{mmol} . \mathrm{g}^{-1}\right.$ at $303 \mathrm{~K}$ and 1 bar $)^{48}$ and SIFSIX-3-Zn (2.3 mmol.g ${ }^{-1}$ at $308 \mathrm{~K}$ and 1 bar $) .{ }^{49}$ However, this $\mathrm{CO}_{2}$ adsorption capacity value is lower than both that of a benchmark zeolite $13 \mathrm{X}\left(5.0 \mathrm{mmol} . \mathrm{g}^{-1} \text { at } 1 \text { bar and } 298 \mathrm{~K}\right)^{50}$ and Mg-MOF-74 (8.0 mmol.g ${ }^{-1}$ at 1 bar and $\left.298 \mathrm{~K}\right)$, although the latter is not water stable. ${ }^{51}$ The initial slope of the $\mathrm{CO}_{2}$ adsorption isotherm is significantly higher than that of $\mathrm{N}_{2}$ and this emphasizes a much stronger affinity of MIL-96(Al) for $\mathrm{CO}_{2}$. Such results are fully consistent with FT-IR, showing a strong adsorption of $\mathrm{CO}_{2}$ at both the Lewis acid $\mathrm{Al}^{3+}$ sites $\left(2345 \mathrm{~cm}^{-1}\right)$ and terminal/bridging hydroxyl groups $\left(2338 \mathrm{~cm}^{-1}\right.$ ) of MIL-96(Al)-HR (see Figure S26). The enthalpy of adsorption for $\mathrm{CO}_{2}$ in MIL-96(Al)-NP2 as a function of loading is given in Figure 7(b). The high experimental enthalpy values at low coverage are certainly due to the interaction of $\mathrm{CO}_{2}$ molecules with $\mathrm{Al}^{3+}$ CUS. Experimental and simulated enthalpy values of $\mathrm{CO}_{2}$ adsorption remain between -35 $\mathrm{kJ} . \mathrm{mol}^{-1}$ and $-30 \mathrm{~kJ} \cdot \mathrm{mol}^{-1}$ throughout the $\mathrm{CO}_{2}$ uptake except at lower coverage. This relatively flat energetic profile indicates a homogeneous energetic interaction with the adsorption sites. 
Interestingly, this adsorption enthalpy for $\mathrm{CO}_{2}$ is substantially higher or comparable to other MOFs (i. e. UiO-66( $\mathrm{Zr}): \sim-26 \mathrm{~kJ} \mathrm{~mol}^{-1}{ }^{52} \mathrm{UiO}-66(\mathrm{Hf})-(\mathrm{OH})_{2}:-28.4 \mathrm{~kJ} \mathrm{~mol}^{-1}{ }^{53}$ and UiO-66(Hf) : -22.8 $\mathrm{kJ} \mathrm{mol}^{-1}{ }^{53}$ however lower than other highly efficient $\mathrm{CO}_{2}$ adsorbents such as zeolite $13 \mathrm{X}(-38 \mathrm{~kJ}$ $\left.\mathrm{mol}^{-1}\right),{ }^{50}$ and MIL-91(Ti) : -43 kJ.mol $\left.{ }^{-1}\right),{ }^{45} \mathrm{MIL}^{-53(\mathrm{Al})-\mathrm{NH}_{2}: \sim-40 \mathrm{~kJ} \cdot \mathrm{mol}^{-1}}{ }^{46,54}, \mathrm{Mg}-\mathrm{MOF}-74(\sim$ $\left.-50 \mathrm{~kJ} . \mathrm{mol}^{-1}\right),{ }^{51,55}$ SIFSIX-3-Zn $\left(-45 \mathrm{~kJ} \cdot \mathrm{mol}^{-1}\right),{ }^{49}$ and SIFSIX-3-Co $\left(-47 \mathrm{~kJ} . \mathrm{mol}^{-1}\right) .{ }^{56}$ Such a moderate adsorption enthalpy suggests an easy reversibility of adsorption-desorption cycles which is highly desirable for the adsorbent regeneration process as a balance between separation performance and energy cost is required in real industrial operations.
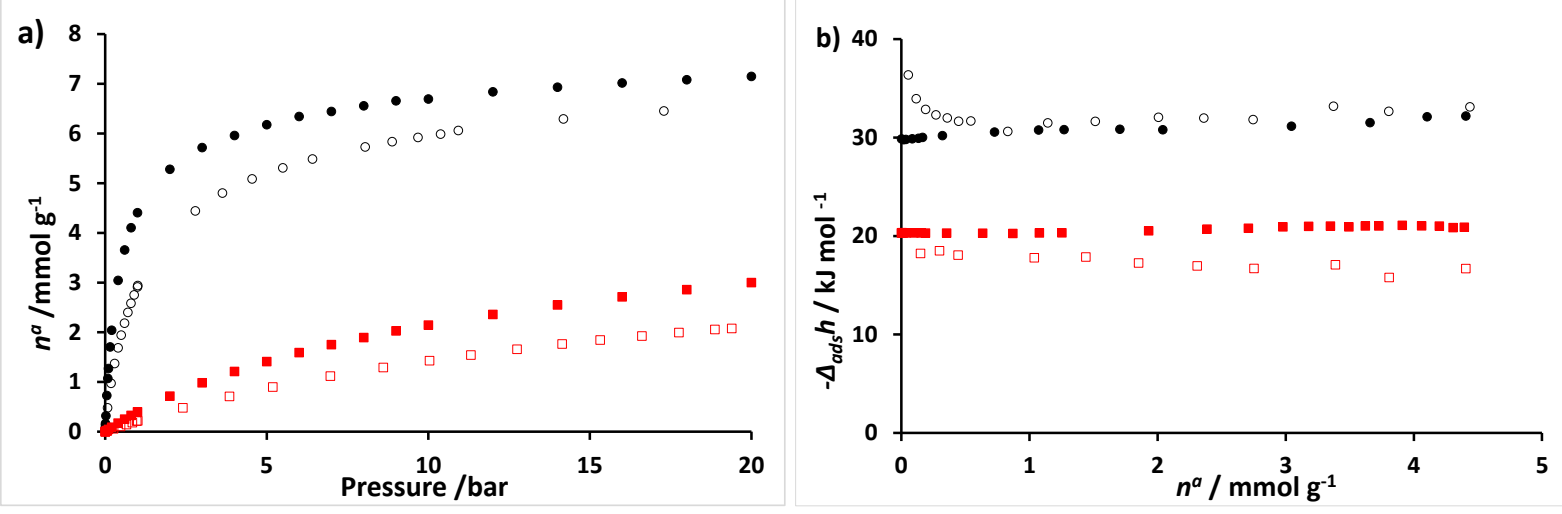

Figure 7. Experimental (empty symbols) and simulated (full symbols) $\mathrm{CO}_{2}$ (black circle) and $\mathrm{N}_{2}$ (red square) single gas adsorption at $303 \mathrm{~K}$ on MIL-96(Al)-NP2 activated at $423 \mathrm{~K}$; (a) adsorption isotherm (i.e amount adsorbed as a function of pressure) and (b) adsorption enthalpies as a function of the coverage collected by microcalorimetry.

Experimental and simulated enthalpy values of $\mathrm{N}_{2}$ adsorption are lower than that of $\mathrm{CO}_{2}$, confirming the much lower affinity of MIL-96(Al) for this guest molecule. However, this value is higher than many medium to large pore MOFs but less than those containing CUS sites, suggesting that the small amount of nitrogen adsorbed is relatively confined, as reported for other MOFs with small pores size $(3.0-4 \cdot 0 \AA) .{ }^{16,45}$

The sorption-based binary gas separation performance of MIL-96(Al)-NP2 was evaluated by considering post-combustion $\mathrm{CO}_{2}$ capture, in which $10 \%$ to $15 \%$ of $\mathrm{CO}_{2}$ needs to be removed from flue gas mixtures containing $\mathrm{N}_{2}$. Co-adsorption measurements were performed on about $10 \mathrm{~g}$ of MIL-96(Al)-NP2. Such real co-adsorption data, rarely reported in the literature, were collected by using an advanced homemade device that combines a volumetric apparatus and gas chromatography analysis (see SI for more details). The measurements were performed at $303 \mathrm{~K}$ for a gas mixture $\mathrm{CO}_{2} / \mathrm{N}_{2}=10 / 90$ and $15 / 85$ at 1.0 bar and 3.0 bar, i.e. the typical industrial concentration and 
pressure conditions for the membrane separation of flue gas emitted from power plants or cement plants. Each mixture point was repeated several times and an average selectivity value was given. The co-adsorption selectivity of $\sim 36$ was obtained at 1 bar and $303 \mathrm{~K}$ for the case of $\mathrm{CO}_{2} / \mathrm{N}_{2}=$ 10/90, and remains nearly constant on increasing the $\mathrm{CO}_{2}$ concentration $\left(\mathrm{CO}_{2} / \mathrm{N}_{2}=15 / 85\right)$. The selectivity values are slightly modified by increasing the pressure to 3 bar (i. e. $S_{\mathrm{EXP}}=29$ for $\left.\mathrm{CO}_{2} / \mathrm{N}_{2}=15 / 85\right)$. Such results are in excellent agreement with data obtained by GCMC simulations (i. e. $\mathrm{S}_{\mathrm{GCMC}}=34$ for $\mathrm{CO}_{2} / \mathrm{N}_{2}=15 / 85$ at 1 bar and $303 \mathrm{~K}$ ) (see Fig. $\mathrm{S} 31$ of $\mathrm{SI}$ ).

\section{Processing of Mixed Matrix Membrane and post-combusion $\mathrm{CO}_{2} / \mathrm{N}_{2}$ separation.}

Due to the excellent colloidal stability of MIL-96(A1)-NP2 and -NP3 in THF, MMMs with a high MOF content (25 wt\%) could be prepared by dispersing both MIL-96(Al)-NP's in a solution of 6FDA-DAM in THF. PXRD of both MIL-96(Al)-NP2/6FDA-DAM and MIL-96(Al)-NP3/6FDADAM MMMs (Figure 8(A)) superimpose well with the one of MIL-96(Al)-NP, confirming that the crystalline structure of MIL-96(Al) is preserved upon its association with 6FDA-DAM. SEM images (Figure 8(B) and Figures S32) of the top-surface of MMMs show the excellent dispersion of MIL-96(Al)-NP2 in the polymer matrix with the absence of any significant aggregation. Such results are confirmed by cross-sectional SEM images (Figures 8 (C, D) and Figure S32), showing a homogeneous distribution of MIL-96(Al) nanocrystals in the volume of the membranes. The thickness of the supported membranes lies between 60 and $65 \mu \mathrm{m}$. Similar results were obtained with MMMs based on MIL-96(Al)-NP3. According to SEM images, no defects or voids of about a few nanometers in size could be observed at the interface between the MIL-96(Al) NP and polymer.

The $\mathrm{CO}_{2}$ adsorption isotherms of membranes with 25 wt \% loading of MIL-96(Al)-NP2 and NP3 in 6FDA-DAM are illustrated in Figure 9(a). To illustrate the possible effect of filler on adsorption properties of MMMs, theoretical isotherms were calculated assuming the additive $\mathrm{CO}_{2}$ adsorption in $25 \mathrm{wt} \% \mathrm{MOF}$ and $75 \mathrm{wt} \%$ polymer (see Figure 9(a)) corresponding to the composition of the MMM. Noteworthy, the experimental $\mathrm{CO}_{2}$ adsorption capacity of MMMs based on MIL-96(Al)-NP2 is lower than the theoretical one, whereas experimental and calculated $\mathrm{CO}_{2}$ 
adsorption are almost similar for MMMs based on MIL-96(Al)-NP3. This is tentatively attributed to a better dispersion of the smaller NP3 particles in the polymer and thus a higher accessibility to gas molecules.
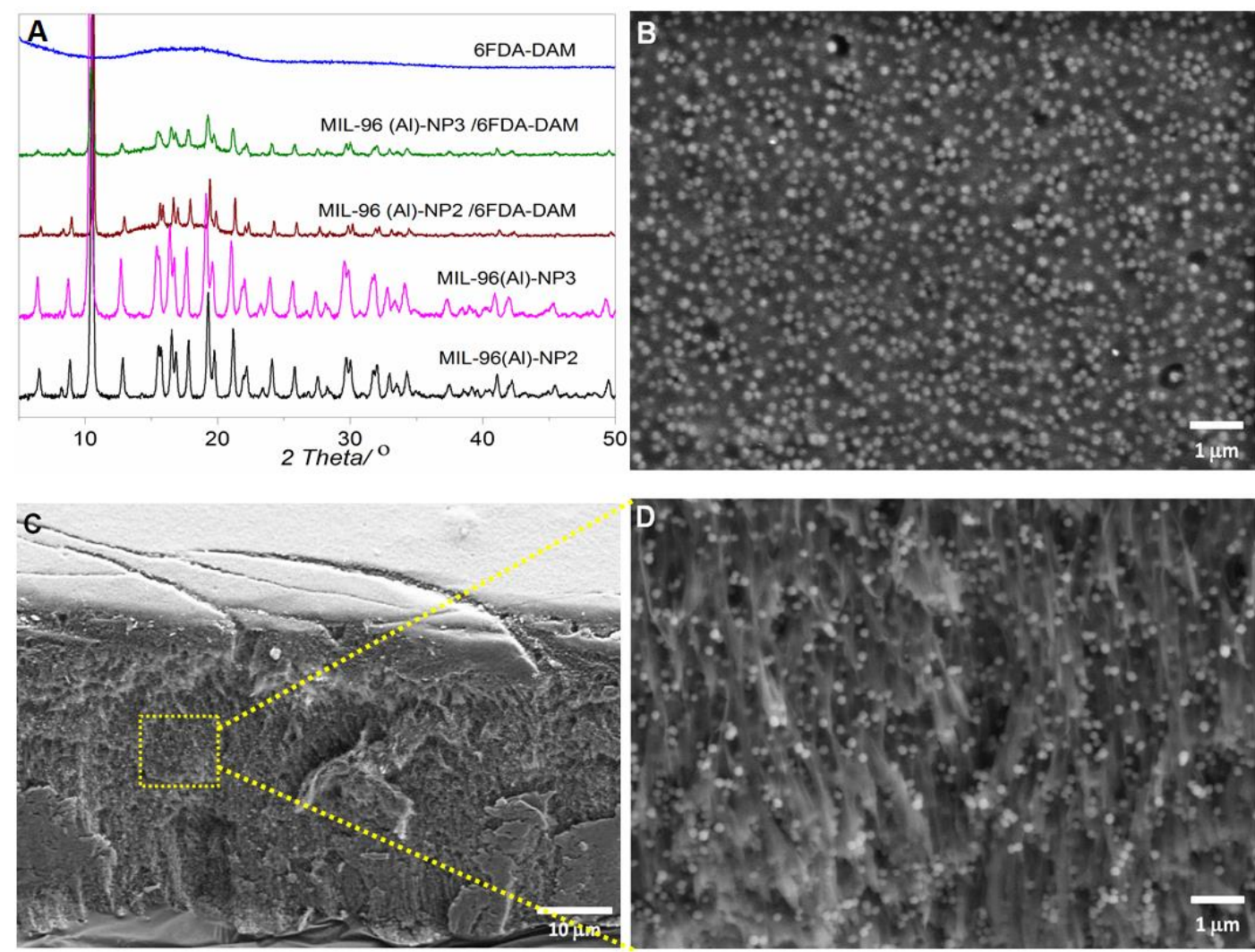

Figure 8. (A) PXRD of the MIL-96(Al)-NP2/6FDA-DAM and MIL-96(Al)-NP3/6FDA-DAM MMMs in comparison with the pure polymer and MIL-96(Al) NP; (B) SEM image of the top-surface of MIL-96(Al)-NP2/6FDA-DAM; (C) cross-section SEM image of MIL-96(Al)-NP2/6FDA-DAM. The high dispersion of MIL-96(Al)-NP2 in the volume of the membrane is confirmed with higher magnification SEM images (D).

The $\mathrm{CO}_{2}$ and $\mathrm{N}_{2}$ separation (15/85 vol\%) at $298 \mathrm{~K}, 2$ bar (abs) feed pressure and helium sweep gas was carried out to study the permeation performance of the MMMs. Remarkably, the addition of MIL-96(A1)-NP2 and -NP3 to the high free volume polymer led to a $\sim 37 \%$ enhancement of the $\mathrm{CO}_{2}$ permeability in 6FDA-DAM, attributed to the high $\mathrm{CO}_{2}$ uptake by MIL-96(Al) (see above). The selectivity improved somewhat as well, slightly more for the smaller particles (Figure (9b)). Moreover, to further examine the performance of MMMs in different feed concentration, the MIL-96(Al)-NP3/6FDA-DAM MMM was tested under 15/85, 50/50 and 85/15 ratios of $\mathrm{CO}_{2} / \mathrm{N}_{2}$ in feed (Table S6). The almost unchanged $\mathrm{CO}_{2} / \mathrm{N}_{2}$ selectivity of MIL-96(Al)-NP2/6FDA-DAM MMM for increased $\mathrm{CO}_{2}$ concentration is in line with the nearly constant selectivity for the $\mathrm{CO}_{2} / \mathrm{N}_{2}$ co-adsorption of pure MOF (see vide supra). Although the permeability of the membrane decreased $(\sim 27 \%)$ with increased $\mathrm{CO}_{2}$ concentration, ascribed to 
saturation of adsorption sites in the membrane matrix, ${ }^{57}$ the $\mathrm{CO}_{2}$ flux through the membrane nevertheless increases with concentration. The influence of the humidity on the $\mathrm{CO}_{2} / \mathrm{N}_{2}$ separation properties of the neat 6FDA-DAM and MIL-96(Al)-NP3/6FDA-DAM membranes was further evaluated (see details in SI). As shown in Table S7, similar results of $\mathrm{CO}_{2} / \mathrm{N}_{2}$ permeability and selectivity were obtained for MIL-96(Al)-NP3/6FDA-DAM membranes exposed to air humidity after two cycles of adsorption/desorption of water. The impact of humidity on the permeation of neat 6FDA-DAM membrane is almost negligible.

Finally, the performance in terms of permeability and selectivity of the MIL-96(Al) based MMMs regarding the Robeson 2008 limit is illustrated in Figure S33. ${ }^{58,59}$ A comparison of their performance with membranes prepared with 6FDA-based polymer is also shown (see Fig. S33 and Table S8). Although the Robeson upper bound is not exceeded, the addition of the filler significantly improved the overall performance of the neat polymeric membrane. Moreover, in comparison to MMMs previously reported, the composite membranes based on MIL-96(Al) present promising performances for post combustion application with enhanced $\mathrm{CO}_{2} / \mathrm{N}_{2}$ selectivity.
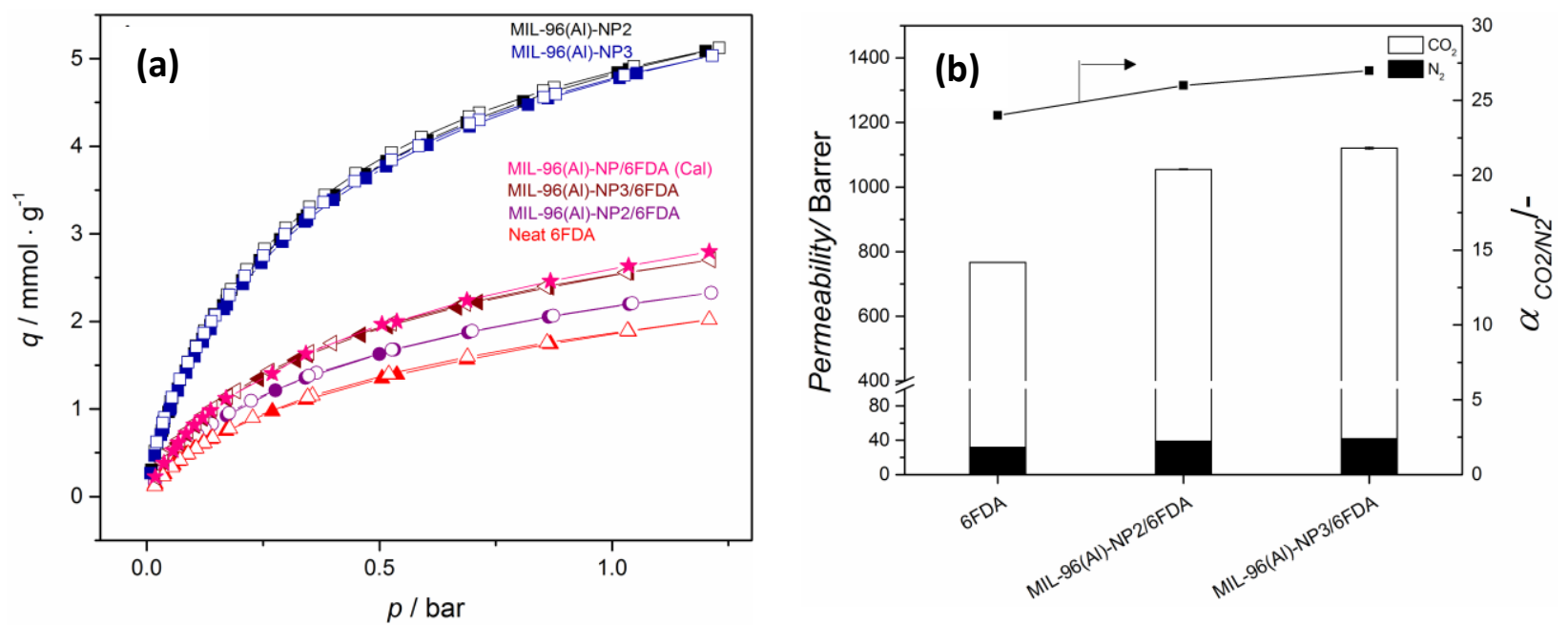

Figure 9. (a) $\mathrm{CO}_{2}$ adsorption isotherms at $273 \mathrm{~K}$ of MIL-96(Al)-NP2 and -NP3, neat 6FDA-DAM, experimental and calculated isotherms of MMMs with $25 \mathrm{wt} \%$. MIL-96(Al)-NP2 and -NP3 loading; (b) Permeation properties for a CO and $\mathrm{N}_{2}$ mixture (15/85) of MMMs comprising 25 wt.\% of MIL-96(Al)-NP2 and -NP3 as filler and 6FDA-DAM as polymer matrix at $298 \mathrm{~K}$ and 2 bars feed pressure. Permeate side atmospheric with helium as sweep gas.

\section{¿ CONCLUSION}

In the present study, by using a wide variety of advanced complementary experimental and computational tools, the crystalline structure of the microporous Al trimesate MIL-96(Al) (anhydrous or hydrated) was reinvestigated allowing a clear description of the local environment of 
$\mathrm{Al}$ sites (symmetry, nature of oxygen ligands). This refinement was required in order to fill gaps in the original structural model reported for MIL-96(Al), ${ }^{15}$ which remained questionable due to inconsistencies between solid state NMR and single crystal XRD experiments. The complex hydrogen bond network formed by the adsorbed water molecules within the MOF framework and its hydroxyl groups was fully described. Several synthesis routes were reported leading pure MIL96(Al) crystals with various morphology and size, down to the nanoscale as required for MMM preparation. Monodisperse MIL-96(Al) nanoparticles were obtained at high yield under reflux conditions and the colloidal solutions present an excellent stability in different solvents (water, THF). The complete characterization of the $\mathrm{CO}_{2} / \mathrm{N}_{2}$ gas sorption properties of MIL-96(Al) including in-situ FT-IR, $\mathrm{CO}_{2} / \mathrm{N}_{2}$ adsorption/co-adsorption experiments, calorimetry and GCMC simulations has shown that MIL-96(Al) presents a high $\mathrm{CO}_{2}$ uptake at low concentration due to the high affinity of $\mathrm{CO}_{2}$ molecules to $\mathrm{Al}^{3+}$ CUS and OH ligands of MIL-96(Al). Finally, MMMs based on MIL-96(Al)-NP and 6FDA-DAM with a high MOF loading ( 25 wt $\%)$ were casted, showing an excellent dispersion of the MOF fillers in the polymer matrix and very promising $\mathrm{CO}_{2} / \mathrm{N}_{2}$ performance (permeability and selectivity). Such properties are likely to be due to the excellent chemical compatibility between MIL-96(Al) and 6FDA-DAM and the absence of any defects or voids at the $\mathrm{MOF} /$ polymer interface. Such results pave the way for the processing of composite membranes with a higher MOF content (>30 wt \%).

\section{ASSOCIATED CONTENT}

Supporting Information. Crystal structure of MIL-96(Al) (CIF); ${ }^{27}$ Al MQMAS NMR and FTIR spectra; TGA; PXRD pattern and structureless refinement of MIL-96(Al) samples; Size distribution of MIL-96(Al)-NPs; Single adsorption isotherms; Simulated $\mathrm{CO}_{2} / \mathrm{N}_{2}$ selectivity; SEM images and $\mathrm{CO}_{2} / \mathrm{N}_{2}$ separation experiments of membranes; This information is available free of charge via the Internet at http://pubs.acs.org/.

\section{a ATHOR INFORMATION}

Corresponding author*Tel.: 331392543 73. E-mail: nathalie.steunou@uvsq.fr. 


\section{- ACKNOWLEDGMENT}

The authors would like to acknowledge the European Community Seventh Program (FP7/20072013) for funding the research presented in this article under Grant Agreement No. 608490 (project M4CO2). They thank the IMPC FR2482 for SEM-FEG instrumentation funded by CNRS, UPMC and C'Nano projects of Région Ile-de-France. They acknowledge synchrotrons SOLEIL (SaintAubin, France) and ALBA (Barcelona, Spain) for SAXS beam time allocation. They thank Thomas

Bizien (SWING, SOLEIL) for his help during SAXS experiments and the staff of the PROXIMA 2A at SOLEIL for technical assistance. G.M. thanks Institut Universitaire de France for its support.

\section{口 REFERENCES}

(1) Themed issue, Chem. Soc. Rev. 2014, 43, 5415-6176.

(2)Themed issue, Chem. Soc. Rev. 2017,46, 3104-3481.

(3) Almeida Paz, F. A.; Klinowski, J.; Vilela, S. M. F.; Tomé, J. P. C.; Cavaleiro, J. A. S.; Rocha, J. Ligand Design for Functional Metal-Organic Frameworks. Chem. Soc. Rev. 2012, 41, 1088-1110.

(4) Padial, N. M.; Quartapelle Procopio, E; Montoro, C.; Lopez, E.; Oltra, J. E.; Colombo, V.; Maspero, A.; Masciocchi, N.; Galli, S.; Senkovska, I.; Kaskel, S.; Barea, E.; Navarro. J. A. R. Highly Hydrophobic Isoreticular Porous Metal-Organic Frameworks for the Capture of Harmful Volatile Organic Compounds. Angew. Chem. Int. Ed. 2013, 52, 8290-8294.

(5) Trickett, C. A.; Helal, Aasif, Al-Maythalony, B. A.; Yamani, Z. H.; Cordova, K. E. ; Yaghi, O. M. The Chemistry of Metal-Organic Frameworks for $\mathrm{CO}_{2}$ Capture, Regeneration and Conversion. Nature Rev. Mater. 2017, 17045.

(6) Cadiau, A.; Belmabkhout, Y.; Adil, K.; Bhatt, P. M.; Pillai, R. S.; Shkurenko, A.; Martineau-Corcos, C.; Maurin, G.; Eddaoudi, M. Hydrolytically Stable Fluorinated Metal-Organic Frameworks for Energy-Efficient Dehydration. Science 2017, 356, 731-735.

(7) Assi, H.; Mouchaham, G.; Steunou, N.; Devic, T.; Serre, C. Titanium Coordination Compounds: from Discrete Metal Complexes to Metal-Organic Frameworks. Chem. Soc. Rev., 2017, 46, 3431-3452.

(8) Maurin, G. Role of Molecular Simulations in the Structure Exploration of Metal-Organic Frameworks : Illustrations through recent advances in the field. C. R. Chimie. 2016, 19, 207-215.

(9) Martineau, C. NMR Crystallography: Applications to Inorganic Materials. Solid State Nucl. Mag. Res. 2014, 63$64,1-12$. 
(10) Bonhomme, C.; Gervais, C.; Babonneau, F. Coelho, C.; Pourpoint, F.; Azaïs, T.; Ashbrook, S. E.; Griffin,J. M.; Yates, J. R.; Mauri, F.; Pickard, C. J. First-Principles Calculation of NMR Parameters Using the Gauge Including Projector Augmented Wave Method: A Chemist's Point of View Chem. Rev. 2012, 112, 5733-5779.

(11) Férey, G.; Mellot-Draznieks, C.; Serre, C.; Millange, F.; Dutour, J.; Surblé, S.; Margilolaki, I. A Chromium Terephthalate-Based Solid with Unusually Large Pore Volumes and Surface Area. Science, 2005, 309, $2040-2042$.

(12) Alvarez, E.; Guillou, N.; Martineau, C.; Bueken, B.; Van de Voorde, B.; Le Guillouzer, C.; Fabry, P.; Nouar, F.; Taulelle, F.; de Vos, D.; Chang, J. -S.; Ho Cho, K.; Ramsahye, N.; Devic, T.; Daturi, M.; Maurin, G.; Serre, S. The Structure of the Aluminum Fumarate Metal-Organic Framework A520. Angew. Chem. Int. Ed. 2015, 54, 36643668 .

(13) Rouquerol, F.; Rouquerol, J.; Sing, K.; Llewellyn, P. L.; Maurin G. In Adsorption by Powders and Porous Solids; Elsevier : Oxford 2nd edn, 2014; Chapter 6, pp. 191-235.

(14) Maurin, G. In The Chemistry of Metal Organic Frameworks : Synthesis, Characterization and Applications;

Kaskel, S. Eds.; Wiley-VCH Verlag GmbH \& Co KGaA: Weinheim, 2016, Volume 2.

(15) Loiseau, T.; Lecroq, L.; Volkringer, C.; Marrot, J.; Férey, G.; Haouas, M.; Taulelle, F.; Bourrelly, S.; Llewellyn, P. L.; Latroche, M. MIL-96, a Porous Aluminum Trimesate 3D Structure Constructed from a Hexagonal Network of 18Membered Rings and $\mu_{3}$-Oxo-Centered Trinuclear Units. J. Am. Chem. Soc. 2006, 128, 10223-10230.

(16) Adil, K.; Belmabkhout, Y.; Pillai, R. S.; Cadiau, A.; Bhatt, P. M.; Assen, A. H.; Maurin, G.; Eddaoudi, M. Gas/vapour Separation using Ultra-microporous Metal-Organic Frameworks: Insights into the Structure/Separation Relationship. Chem. Soc. Rev., 2017, 46, 3402-3430.

(17) Maes, M.; Alaerts, L.; Vermoortele, F.; Ameloot, R.; Couck, S.; Finsy, V.; Denayer, J. F. M.; De Vos, D. E. Separation of C5-Hydrocarbons on Microporous Materials: Complementary Performance of MOFs and Zeolites. J. Am. Chem. Soc. 2010, 132, 2284-2292.

(18) Knebel, A.; Friebe, S.; Bigall, N. C.; Benzaqui, M.; Serre, C.; Caro, J. Comparative Study of MIL-96(Al) as Continuous Metal-Organic Frameworks Layer and Mixed-Matrix Membrane. ACS Appl. Mater. Interfaces 2016, 8, 7536-7544.

(19) Qiu, M.; Chen, C.; Li, W. Rapid Controllable Synthesis of Al-MIL-96 and Its Adsorption of Nitrogenous VOCs. Catal. Today 2015, 258, 132-138.

(20) Lan, X.; Zhang, H.; Bai, P.; Guo, X. Investigation of Metal Organic Frameworks for the Adsorptive Removal of Hydrochloride from Dilute Aqueous Solution. Microporous Mesoporous Mater. 2016, 231, 40-46.

(21) Zhang, N.; Yang, X.; Yu, X.; Jia, Y.; Wang, J.; Kong, L.; Jin, Z.; Sun, B.; Luo, T.; Liu, J. Al-1,3,5benzenetricarboxylic Metal-Organic Frameworks : A Promising Adsorbent for Defluoridation of Water with pH Insensitivity and Low Aluminum Residual. Chem. Eng. J. 2014, 252, 220-229. 
(22) Zornoza, B.; Tellez, C.; Coronas, J.; Gascon, J.; Kapteijn, F. Metal Organic Framework based Mixed Matrix Membranes: An Increasingly Important Field of Research with a Large Application Potential. Microporous Mesoporous Mater. 2013, 166, 67-78.

(23) Seoane, B.; Coronas, J.; Gascon, I.; Benavides, M. E.; Karvan, O.; Caro, J.; Kapteijn, F.; Gascon, J. MetalOrganic Framework based Mixed Matrix Membranes: a Solution for Highly Efficient $\mathrm{CO}_{2}$ Capture? Chem. Soc. Rev. $\mathbf{2 0 1 5}, 44,2421-2454$.

(24) Qiu, S.; Xue, M.; Zhu, G. Metal-Organic Framework Membranes: from Synthesis to Separation Application. Chem. Soc. Rev. 2014, 43, 6116-6140.

(25 ) Zhang, Y.; Feng, X.; Yuan, S.; Zhou, J.; Wang, B. Challenges and Recent Advances in MOF-polymer Composite Membranes for Gas Separation. Inorg. Chem. Front. 2016, 3, 896-909.

(26) Denny, Jr., M. S.; Moreton, J. C. ; Benz, L.; Cohen, S. M. Metal-organic Frameworks for Membrane-based Separations. Nature Rev. Mater. 2016, 16078.

(27) Perez, E.V.; Balkus Jr., K. J.; Ferraris, J. P.; Musselman, I. H. Mixed-Matrix Membranes Containing MOF-5 for Gas Separations. J. Membr. Sci. 2009, 328, 165-173.

(28) Ordonez, M. J. C.; Balkus Jr, K. J.; Ferraris, J. P.; Musselman, I. H.; Molecular Sieving Realized with ZIF8/Matrimid(R) Mixed-Matrix Membranes. J. Membr. Sci. 2010, 361, 28-37.

(29) Benzaqui, M.; Semino, R.; Menguy, N.; Carn, F.; Kundu,T.; Guigner, J.-M.; McKeown, N. B.; Msayib, K. J.; Carta, M.; Malpass-Evans, R.; Le Guillouzer, C.; Clet, G.; Ramsahye, N. A.; Serre, C.; Maurin, G.; Steunou, N. Toward an Understanding of the Microstructure and Interfacial Properties of PIMs/ZIF-8 Mixed Matrix Membranes. ACS Appl. Mater. Interfaces 2016, 8, 27311-27321.

(30) Khan, N. A.; Lee, J. S.; Jeon, J.; Jun, C.-H.; Jhung, S. H. Phase-Selective Synthesis and Phase-Conversion of Porous Aluminum-Benzenetricarboxylates with Microwave Irradiation.Microporous Mesoporous Mater. 2012, 152, 235-239.

(31) Haouas, M.; Volkringer, C.; Loiseau, T.; Férey, G.; Taulelle, F. In Situ NMR, Ex Situ XRD and SEM Study of the Hydrothermal Crystallization of Nanoporous Aluminum Trimesates MIL-96, MIL-100, and MIL-110. Chem. Mater. 2012, 24, 2462-2471.

(32) Seoane, B.; Dikhtiarenko, A.; Mayoral, A.; Tellez, C.; Coronas, J.; Kapteijn, F.; Gascon, J. Metal Organic Framework Synthesis in the Presence of Surfactants: Toward Hierarchical MOFs? CrystEngComm 2015, 17, 16931700.

(33) Qiu, M.; Guan, Q.; Li, W. Controllable Assembly of Al-MIL-100 via an Inducing Occupied effect and Its Selective Adsorption Activity. Cryst. Growth Des. 2016, 16, 3639-3646. 
(34) Sindoro, M.; Jee, A.-Y.; Granick, S. Shape-Selected Colloidal MOF Crystals for Aqueous Use. Chem. Commun. 2013, 49, 9576-9578.

(35) Liu, D.; Liu, Y.; Dai, F.; Zhao, J.; Yang, K.; Liu, C. Size- and Morphology-Controllable Synthesis of MIL-96(Al) by Hydrolysis and Coordination Modulation of Dual Aluminum Source and Ligand Systems. Dalton Trans. 2015, 44, 16421-16429.

(36) Reinsch, H. ; Stock, N. High-throughput Studies of Highly Porous Al-based MOFs. Microporous Mesoporous Mater. 2013, 171, 156-165.

(37) Zahn, G.; Zerner, P.; Lippke, J.; Kempf, F. L.; Lilienthal, S.; Schröder, C. A.; Schneider, A. M.; Behrens, P. Insight into the Mechanism of Modulated Syntheses: in situ Synchrotron Diffraction Studies on the Formation of $\mathrm{Zr}$-fumarate MOF. CrystEngComm. 2014, 16, 9198-9207.

(38) Schaate, A.; Roy, P.; Godt, A.; Lippke, J.; Waltz, F.; Wiebcke, M.; Behrens, P. Modulated Synthesis of Zr-Based Metal-Organic Frameworks: From Nano to Single Crystals. Chem. - Eur. J. 2011, 17 (24), 6643-6651.

(39) Leclerc, H.; Vimont, A.; Lavalley, J.-C.; Daturi, M.; Wiersum, A. D.; Llewellyn, P. L.; Horcajada, P.; Férey, G.; Serre, C. Infrared Study of the Influence of Reducible Iron(III) Metal Sites on the Adsorption of $\mathrm{CO}, \mathrm{CO}_{2}$, Propane, Propene and Propyne in the Mesoporous Metal-Organic Framework MIL-100. Phys. Chem. Chem. Phys. 2011, 13, $11748-11756$.

(40) Lieb, A.; Leclerc, H.; Devic, T.; Serre, C.; Margiolaki, I.; Mahjoubi, F.; Lee, J. S.; Vimont, A.; Daturi, M.; Chang, J.-S. MIL-100(V) - A Mesoporous Vanadium Metal Organic Framework with Accessible Metal Sites. Microporous Mesoporous Mater. 2012, 157, 18-23.

(41) Hadjiivanov, K. Identification and Characterization of Surface Hydroxyl Groups by Infrared Spectroscopy. Adv. Catal. 2014, 57, 99-318.

(42) Hadjiivanov, K.; Vayssilov, G. Characterization of Oxide Surfaces and Zeolites by Carbon Monoxide as an IR Probe Molecule. Adv. Catal. 2002, 47, 307-511.

(43) Mihaylov, M.; Chakarova, K.; Andonova, S.; Drenchev, N.; Ivanova, E.; Pidko, E. A.; Sabetghadam, A.; Seoane, B.; Gascon, J.; Kapteijn, F.; K. Hadjiivanov, K. Adsorption of $\mathrm{CO}_{2}$ on MIL-53(Al): FTIR Evidence of the Formation of Dimeric $\mathrm{CO}_{2}$ Species. Chem. Commun. 2016, 52, 1494-1497.

(44) Serre, C.; Bourrelly, S.; Vimont, A.; Ramsahye, N. A.; Maurin, G.; Llewellyn, P. L.; Daturi, M.; Filinchuk, Y.; Leynaud, O.; Barnes, P.; Férey, G. An Explanation for the Very Large Breathing Effect of a Metal-Organic Framework during $\mathrm{CO}_{2}$ Adsorption. Adv. Mater. 2007, 19, 2246-2251.

(45) Benoit, V.; Pillai, R. S.; Orsi, A.; Normand, P.; Jobic, H.; Nouar, F.; Billemont, P.; Bloch, E.; Bourrelly, S.; Devic, T.; Wright, P. A.; Weireld, G. de; Serre, C.; Maurin, G.; Llewellyn, P. L. MIL-91(Ti), a Small Pore Metal-organic 
Framework Which Fulfils Several Criteria: An Upscaled Green Synthesis, Excellent Water Stability, $\mathrm{High}_{\mathrm{CO}}$ Selectivity and Fast $\mathrm{CO}_{2}$ Transport. J. Mater. Chem. A 2016, 4, 1383-1389.

(46) Stavitski, E.; Pidko, E. A.; Couck, S.; Remy, T.; Hensen, E. J. M.; Weckhuysen, B. M.; Denayer, J.; Gascon, J.; Kapteijn, F. Complexity behind $\mathrm{CO}_{2}$ Capture on $\mathrm{NH}_{2}-\mathrm{MIL}-53(\mathrm{Al})$. Langmuir 2011, 27, 3970-3976.

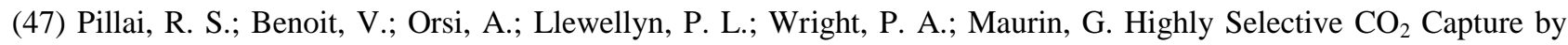
Small Pore Scandium-Based Metal-Organic Frameworks. J. Phys. Chem. C 2015, 119, 23592-23598.

(48) Yang, Q.; Vaesen, S.; Ragon, F.; Wiersum, A. D.; Wu, D.; Lago, A.; Devic, T.; Martineau, C.; Taulelle, F.; Llewellyn, P. L.; Jobic, H.; Zhong, C.; Serre, C.; De Weireld, G.; Maurin, G. A Water Stable Metal-Organic Framework with Optimal Features for $\mathrm{CO}_{2}$ Capture. Angew. Chem. Int. Ed. 2013, 52, 10316-10320.

(49) Nugent, P.; Belmabkhout, Y.; Burd, S. D.; Cairns, A. J.; Luebke, R.; Forrest, K.; Pham, T.; Ma, S.; Space, B.; Wojtas, L.; Eddaoudi, M.; Zaworotko, M. J. Porous Materials with Optimal Adsorption Thermodynamics and Kinetics for $\mathrm{CO}_{2}$ Separation. Nature 2013, 495, 80-84.

(50) Cavenati, S.; Grande, C. A.; Rodrigues, A. E. Adsorption Equilibrium of Methane, Carbon Dioxide, and Nitrogen on Zeolite 13X at High Pressures. J. Chem. Eng. Data 2004, 49, 1095-1101.

(51) Caskey, S. R.; Wong-Foy, A. G.; Matzger, A. J. Dramatic Tuning of Carbon Dioxide Uptake via Metal Substitution in a Coordination Polymer with Cylindrical Pores. J. Am. Chem. Soc. 2008, 130, 10870-10871.

(52) Yang, Q.; Wiersum, A. D.; Llewellyn, P. L.; Guillerm, V.; Serre, C.; Maurin, G. Functionalizing Porous Zirconium Terephthalate UiO-66(Zr) for Natural Gas Upgrading: a Computational Exploration. Chem. Commun. 2011, 47, 96039605 .

(53) Hu, Z.; Nalaparaju, A.; Peng, Y.; Jiang, J.; Zhao, D. Modulated Hydrothermal Synthesis of UiO-66(Hf)-Type Metal-Organic Frameworks for Optimal Carbon Dioxide Separation. Inorg. Chem. 2016, 55, 1134-1141

(54) Serra-Crespo, P.; Berger, R.; Yang, W.; Gascon, J.; Kapteijn, F., Separation of $\mathrm{CO}_{2} / \mathrm{CH}_{4}$ Mixtures over $\mathrm{NH}_{2}-\mathrm{MIL}-$ 53-An Experimental and Modelling Study. Chem. Eng. Sci. 2015, 124, 96-108.

(55) Britt, D.; Furukawa, H.; Wang, B.; Glover, T. G.; Yaghi, O. M. Highly Efficient Separation of Carbon Dioxide by a Metal-Organic Framework Replete with Open Metal Sites. Proc. Natl. Acad. Sci. U. S. A. 2009, 106, $20637-20640$.

(56) Elsaidi, S. K.; Mohamed, M. H.; Schaef, H. T.; Kumar, A.; Lusi, M.; Pham, T.; Forrest, K. A.; Space, B.; Xu, W.; Halder, G. J.; Liu, J.; Zaworotko, M. J. Thallapally, P. K. Hydrophobic Pillared Square Grids for Selective Removal of $\mathrm{CO}_{2}$ from Simulated Flue Gas. Chem. Commun. 2015, 51, 15530-15533.

(57) Paul, D. R. Gas Sorption and Transport in Glassy Polymers. Berich. Bunsen. Gesell.1979, 83, $294-302$.

(58) Robeson, L. M. Correlation of Separation Factor Versus Permeability for Polymeric Membranes. J. Membr. Sci. 1991, 62, 165-185.

(59) Robeson, L. M. The Upper Bound Revisited. J. Membr. Sci. 2008, 320, 390-400. 
Table of Contents

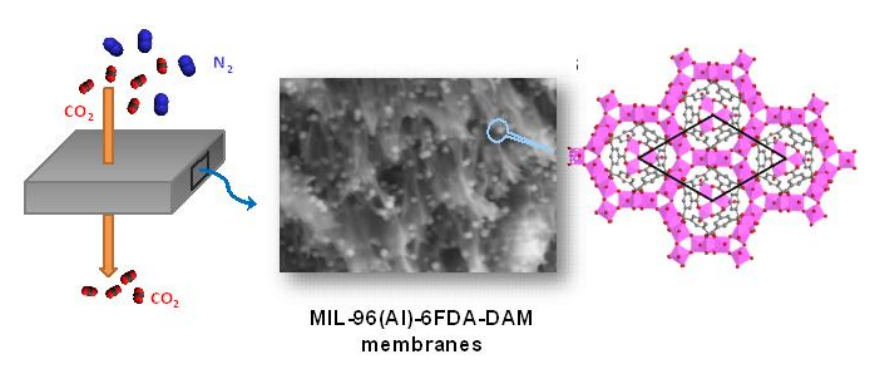

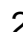

3

4

5

6
7

8

9

10

12

13

14

15

17

18

19

20

21

22

23

24

25

26

27

28

29

30

31

32

33

34

35

36

37

38

39

40

41

42

43

44

45

46

47

48

49

50

51

52

53

54

55

56

57

58

59

60 